\title{
SURVEY ON NEW TECHNIQUES FOR X-RAY ASTRONOMY
}

\author{
RICCARDO GIACCONI \\ American Science and Engineering, Inc., Cambridge, Mass., U.S.A.
}

\begin{abstract}
The limitations imposed in X-ray astronomy observations by the use of sounding rockets with their inherent short time for observations and restricted payload capabilities will soon be surmounted when the new generation of X-ray experiments carried aloft by earth orbitting satellites is launched.

In the early 1970's several payloads included in the spacecrafts SASA, OSOH, SAS C, OSO 1, UK 5 and ANS will perform high sensitivity surveys of the entire celestial sphere. As a result of these experiments we expect to: increase the sensitivity of our observations by a factor of approximately 100 ; to improve the measurement of location on all known sources and on newly discovered ones to an accuracy of $\frac{1}{2}$ to $6 \mathrm{arc} \min$; to acquire more definite information on rapid time variations of the $\mathrm{X}$-ray sources; to measure the polarization of the incoming $\mathrm{X}$-ray radiation to several per cent for the strongest sources; and, finally, to determine for the strongest sources the existence of emission lines or absorption edges to the accuracy required to confirm or disprove present theories of source of emission processes and interstellar absorption.

Beyond these surveys a new generation of X-ray instrumentation utilizing focussing X-ray telescopes is in the planning stage. These instruments will yield a qualitative advance in the type of observations that can be performed. Location and structure of X-ray sources can be determined with these techniques to precisions of a few arc seconds to a limiting surface brightness of $10^{-14} \mathrm{erg} / \mathrm{cm}^{2} \mathrm{~s}$ $\left(\operatorname{arc} s^{2}\right)$. Spectroscopy of the strongest sources can be carried out with energy resolutions $\lambda / \Delta \lambda$ of $10^{3}$ to $10^{4}$. Polarization measurements can be carried out to a few per cent on the Crab and rapid varying events can be followed with a time resolution of $10^{-4} \mathrm{~s}$.

A summary of the instrumentation will be given from the point of view of the developments and improvements which are being carried out to achieve the observational goals mentioned above.
\end{abstract}

A review of the status of knowledge in X-ray astronomy in the eight years since the discovery of cosmic X-ray sources, reveals a very rewarding period of survey and discovery which has proceeded in concert with a phase of rapid technical development.

The observations have in the main been carried out by use of rockets in the regions between 0.25 to $25 \mathrm{keV}$ and from balloons at higher energies. At the low energies the net X-ray exposure, defined as available detector area $A$ times the observations time $t$ in $\mathrm{cm}^{2} \mathrm{~s}$, that can be achieved by the rocket carriers, is of the order of $3 \times 10^{5} \mathrm{~cm}^{2} \mathrm{~s}$. Although this represents an increase of about $10^{2}$ on the first stellar X-ray payloads, it severely limits the sensitivity of the measurements which can be carried out. Also, since most of the measurements to date have been noise rather than signal limited, the improvement in sensitivity is related to the square root of At rather than to its first power. In balloon experiments exposure as high as $10^{8} \mathrm{~cm}^{2} \mathrm{~s}$ have been obtained. However, if one considers that one is attempting to obtain photon statistics at higher energy from sources which exhibit rapid decreasing fluxes with increasing energy and in the presence of an increasingly severe background, it is not surprising to find that in all cases new sources have been observed by rockets rather than by balloons.

These limitations have prompted very intensive efforts to reduce the sources of background counts in the detectors; efforts which have met, as we shall see, with considerable success. However, even with these improvements, we are barely able to detect the 
strongest of the extragalactic sources and, we suspect, only certain classes of galactic objects. Also we are not in the position to utilize fully any of the refined techniques of $\mathrm{X}$-ray imaging, spectrometry or polarimetry to investigate the detailed characteristics of the observed radiation.

In the very near future, however, this situation will radically change when a new generation of X-ray experiments carried aloft by earth orbiting satellites is launched. Individually these experiments are relatively modest. Taken together, however, the increased exposure times will not only considerably extend the sensitivity of all-sky surveys, but also, and perhaps most importantly, they will permit the use of spectrum analysers and polarimeters with the expectation of statistically significant measurements. In a satellite, such as SAS A, to be flown late this year, the exposure time will be of the order of $10^{10} \mathrm{~cm}^{2} \mathrm{~s}$, or about $3 \times 10^{4}$ greater than in rockets. Thus, for identical instruments carried on SAS A and on a rocket, one can expect an improvement of sensitivity of more than two orders of magnitude. The spacecrafts and experiments presently under development are listed in Table I. The effective detector area of the instruments is typically of several hundred $\mathrm{cm}^{2}$. Fields of view range from $\frac{1}{2}^{\circ}$ to ten's of degrees; however, arc minute angular resolution will be achieved through use of modulation collimators. The later payloads include polarimeters and Bragg crystal spectrometers.

As a result of these experiments, we expect to increase the sensitivity of our observations by a factor of about 100 , as mentioned above; to improve the measurement of location of all known sources and newly discovered ones to an accuracy of $\frac{1}{2}$ to 10 arc

\section{TABLE I}

\begin{tabular}{|c|c|c|c|c|c|}
\hline Spacecraft & Year & Institutions & Mode & Energy range & Objectives \\
\hline SAS A & 1970 & AS\&E & Spinning & Medium & $\begin{array}{l}\text { All sky survey } \\
\text { Source Locations } \\
\text { Simultaneous Observations }\end{array}$ \\
\hline $\mathrm{OSO} \mathrm{H}$ & 1971 & MIT & Spinning & Med-High & $\begin{array}{l}\text { All sky survey } \\
\text { Non-dispersive spectra }\end{array}$ \\
\hline SAS C & 1973 & MIT & $\begin{array}{l}\text { Spinning } \\
\text { Pointing }\end{array}$ & Low-Med & $\begin{array}{l}\text { Restricted sky survey } \\
\text { Source Locations } \\
\text { High Time resolution }\end{array}$ \\
\hline OSO 1 & 1973 & $\begin{array}{l}\text { GSFC } \\
\text { Wisc. } \\
\text { Columbia }\end{array}$ & $\begin{array}{l}\text { Pointing } \\
\text { Pointing } \\
\text { Pointing } \\
\text { Scanning }\end{array}$ & $\begin{array}{l}\text { Med } \\
\text { Low } \\
\text { Med } \\
\text { Med }\end{array}$ & $\begin{array}{l}\text { Background survey } \\
\text { Soft X-ray survey } \\
\text { Polarimetry } \\
\text { Mosaic crystal spectrometer }\end{array}$ \\
\hline UK 5 & 1973 & $\begin{array}{l}\text { Leicester } \\
\text { U.C., London } \\
\text { U.C., London } \\
\text { Leicester }\end{array}$ & $\begin{array}{l}\text { Scanning } \\
\text { Pointing } \\
\text { Pointing } \\
\text { Pointing }\end{array}$ & $\begin{array}{l}\text { Med } \\
\text { Low-Med } \\
\text { Med } \\
\text { Med }\end{array}$ & $\begin{array}{l}\text { All sky survey } \\
\text { Source Locations } \\
\text { Non-dispersive spectra } \\
\text { Polarimetry, Crystal } \\
\text { spectrometry }\end{array}$ \\
\hline & & Imperial Col. & Pointing & High & $\begin{array}{l}\text { Scintillation counter } \\
\text { spectrometry }\end{array}$ \\
\hline ANS & 1974 & $\begin{array}{l}\text { Utrecht } \\
\text { ASE-MIT }\end{array}$ & $\begin{array}{l}3 \text {-axis point } \\
\text { 3-axis point }\end{array}$ & $\begin{array}{l}\text { Low } \\
\text { Med }\end{array}$ & $\begin{array}{l}\text { Source background spectrum } \\
\text { Bragg crystal spectrometer }\end{array}$ \\
\hline
\end{tabular}


min; to acquire more definite information on rapid time variations of the X-ray sources; to measure the polarization of the incoming X-ray radiation to a few per cent for the strongest sources; and, finally, to determine for the strongest sources the existence of emission lines or absorption edges to the accuracy required to confirm or disprove present theories of source emission processes and interstellar absorption.

It would be impossible to describe in detail each of the instrumented payloads for these missions, particularly since several are just now in the design phase. Rather one can briefly touch on the technical developments which have occurred in the various areas of X-ray instrumentation. These developments have been partly encouraged by the opportunities now opened for more refined instruments to be placed on these satellites, partly by the fact that while these 'conventional' satellite payloads are being built (with execution times of years, intrinsic in all space missions), a new generation of rockets payloads is being developed which will provide much of the exploratory work in new regions of the spectrum. These payloads will also eventually be flown on the satellite carriers which are being planned for the 1975 to 1980 period.

In dealing with each element of X-ray instrumentation I will only attempt to describe the improvements which have occurred in the last few years and to show how these techniques are integrated in one or two typical experiments. For a more detailed description of the basic components, I would refer to a number of reviews in the field which have appeared in the last few years. (de Jager, 1967; Giacconi et al., 1968b, Giacconi et al.,1969; Gursky, 1970).

\section{Collimators}

Mechanical baffles placed in front of broad field of view X-ray detectors have been extensively used to precisely define the desired angle of acceptance. Collimator arrays extending over areas as large as $1000 \mathrm{~cm}^{2}$ with $80 \%$ transmission and with a high degree of alignment have been built. A collimator of $\frac{1}{2}^{\circ} \times 5^{\circ} \mathrm{FWHM}$ will be utilized on SAS A. The angular response of the device is shown in Figure 1, and is sufficiently precise and stable that accuracies of peak location as fine as a few minutes of arc are expected for strong sources (Giacconi et al., 1968). Except for fabrication techniques, no significant development has occurred in recent years, although several laboratories are considering the problems involved in maintaining the desired degree of coalignment over areas of several square feet.

Multi-grid modulation collimators first suggested by Oda (1965) have been used by the MIT group in a somewhat novel fashion in a recent rocket launch (October, 1969). A principal difficulty for use of modulation collimators is due to the reduced sensitivity one can achieve because of the loss of effective area and time on source. Thus, after the initial use in the case of Sco X-1 and Crab (Gursky et al., 1966; Oda et al., 1967), one had to wait for the development of background reduction techniques to use the device on weaker sources. A second difficulty is that when several sources are simultaneously within the field of view the intrinsic ambiguities of the response become extremely difficult to resolve. The MIT group used a particular scan mode which 
consists of rotating the modulation collimator along its axis as suggested by Mertz (Mertz, 1967). The effect of this motion can be seen in Figure 1. Conceptually, if one keeps the modulation collimator stationary and rotates the source in the sky, which is equivalent, it is obvious that the signal from each source in the field of view is modulated by a frequency which is fixed by the radial distance from the intersection of the rotation axis and the celestial sphere. The phase of the modulation is determined

(a)

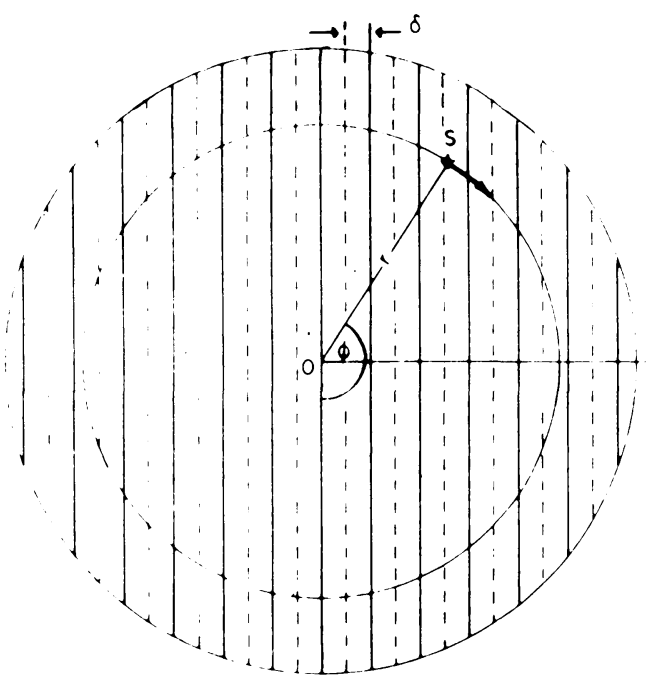

(b)

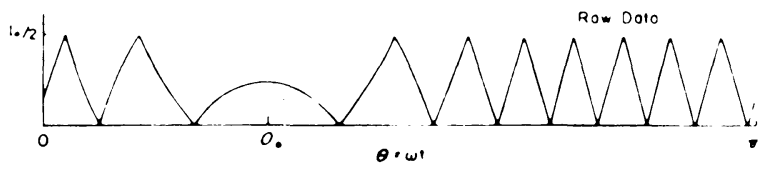

Fig. 1. Schematic representation of the operation of a rotating modulation collimator. The solid lines in part (a) of the figure represent the view direction of a modulation collimator rotating around a point $\mathrm{O}$ in the sky. A source $s$ at an angular distance $r$ from the center of rotation will modulate as shown in part (b) of the figure assuming infinite statistics and constant rotating speed.

by the angular distance of the source from a reference line on the celestial sphere. Thus, the modulation function for each source is unique. Schnopper, Thompson and Watt, of MIT (Schnopper et al., 1968), worked out an analysis technique based on Fourier analysis and were able to show that this method has the ability to distinguish and accurately locate each source in a multiple source field. An experiment was carried out by the MIT group utilizing this technique (Schnopper et al., 1970) and obtained the position of several X-ray sources to \pm 30 arc sec. The results are shown 


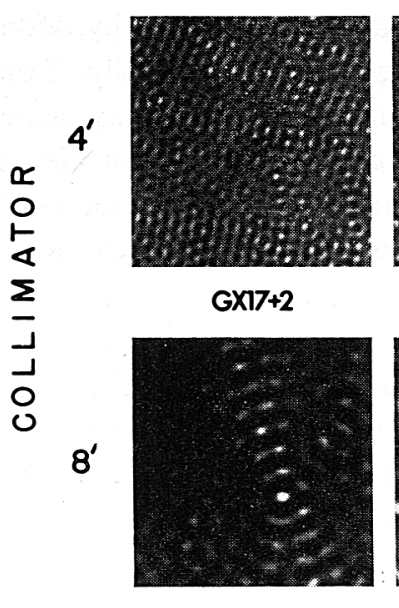

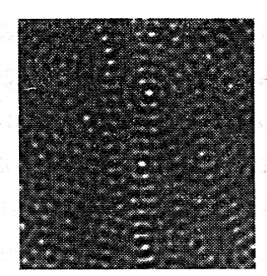

GX9+1

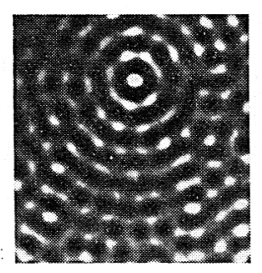

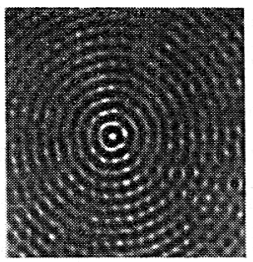

GX5-1

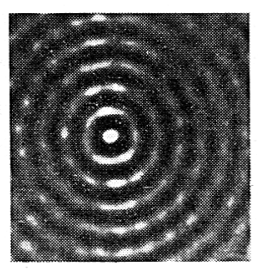

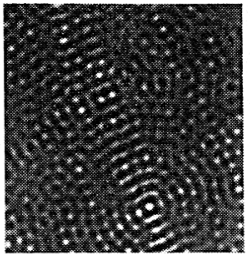

GX3+1

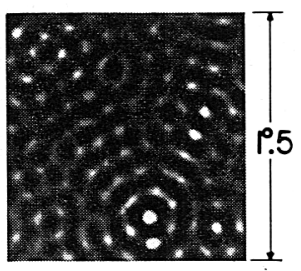

$1^{\circ} .5$

Fig. 2. A graphic representation of the results obtained during the October 1969 rocket flight by Schnopper et al. The white regions in the figure indicate regions where the data have high correlation with the response expected from a point source at the location. The ring structures are typical of the response to a bright source. (Courtesy H. Schnopper, MIT group).

in graphic form in Figure 2. The white regions indicate points where the data have a high correlation with the response expected for a source at that point in the sky.

\section{Focusing Collectors (Non-imaging)}

Focusing collectors for use in X-ray astronomy were suggested as early as 1960 by Giacconi and Rossi, who described a number of possible configurations for concentrating the X-rays entering a large aperture device into a small focal spot (Giacconi and Rossi, 1960; Giacconi and Rossi, 1961). The collectors use the fact that X-rays reflect with high efficiency at grazing incidence. Figure 3 shows computed efficiencies of reflection for X-rays at different wavelengths from a variety of surfaces. The use of collectors in recent years has been stimulated by the interest in extending the X-ray surveys at long wavelengths. Since the critical angle of grazing incidence $\theta_{c} \sim \lambda$ increases with increasing wavelength, the construction and use of collectors with substantial collecting areas is simplified at long wavelength, 10 to $60 \AA$. Several systems have been constructed in recent years. Fisher (Fisher and Meyerott, 1966) described in 1965 the realization of a device based on segments of flat mirrors approximating a paraboloidal one dimensional collector. More recently, our group at AS\&E has implemented an experiment utilizing a one-dimensional focusing collector with mirrors of parabolic cross section, which was flown for a survey of Virgo and Cygnus in the 10 to $60 \AA$ region on June 26,1970 . The payload will be described in detail by P. Gorenstein in a separate contribution to this meeting (Gorenstein et al., 1970). The principle of the device is illustrated in Figure 4. The advantages offered by the device are (a) the exclusion of low energy charged particles which contribute significantly 


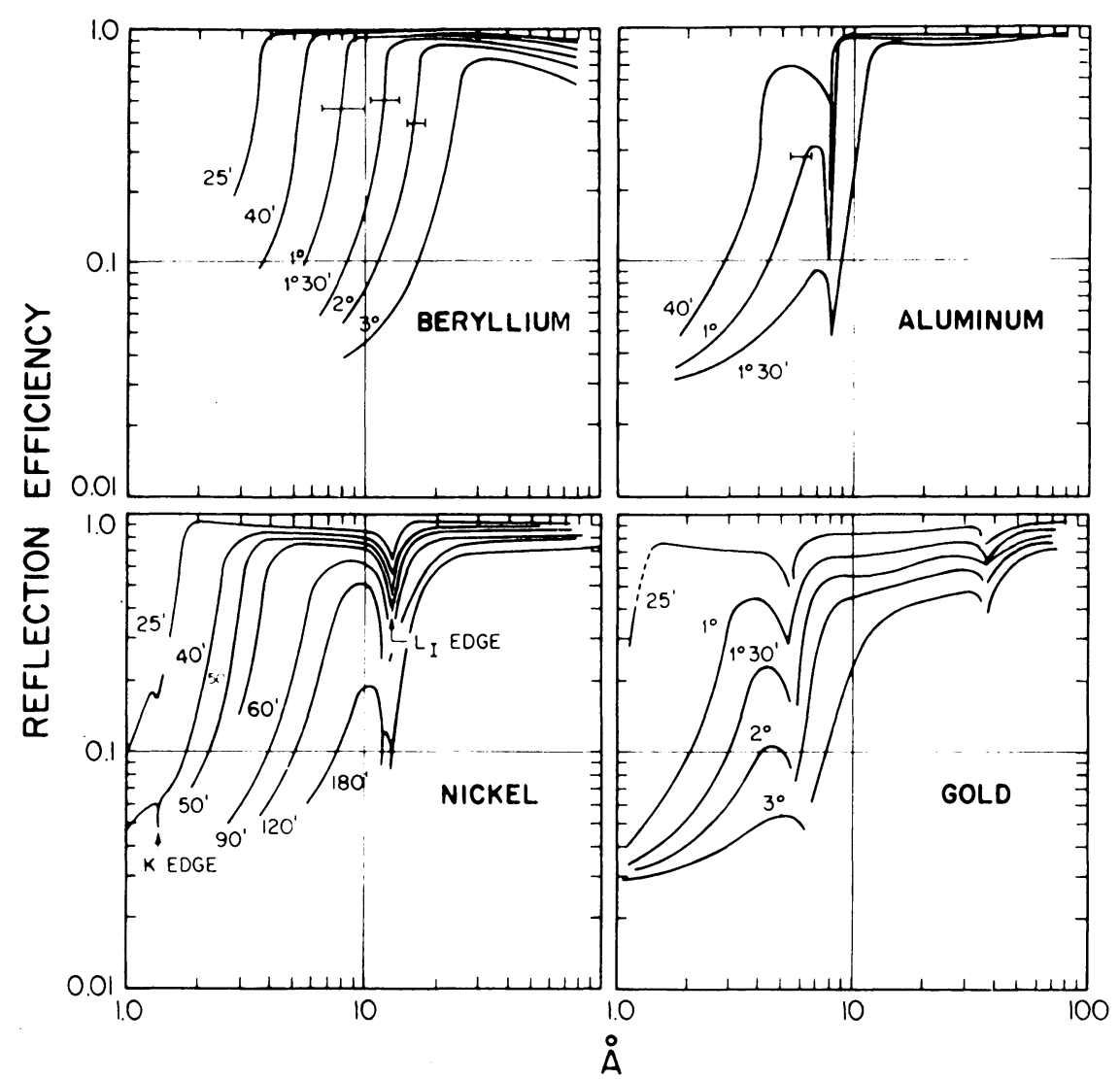

Fig. 3. Theoretical reflection efficiency vs. wavelength for various grazing angles and reflection materials.

to the background particularly when very thin window proportional counters are used, and (b) a gain in the signal-to-noise ratio is achieved which is proportional to the ratio of the area of the collector to the area of the resolution element in the focal plane. With practical detectors and focal lengths for use in rocket work improvements in the signal-to-noise ratio of the order of 10 to 100 can be achieved. An advanced version of the payload developed by the Lockheed group will be described by Fisher at this meeting.

Similar considerations apply to the collector used by Columbia University in their recent flight, devoted to a survey of the soft component of the background in the region between 0.1 and $0.3 \mathrm{keV}$. This rocket payload will also be described in detail by the Columbia group at this meeting and is shown conceptually in Figure 5. There is a significant difference in the two focusing systems, namely that it is not possible in the case of the parabolic collector to map the celestial sphere unambiguously in the focal plane by use of simple detectors. Thus, once a desired angular 
resolution $\alpha$ is defined a single detector in the focal plane of radius $r, r=f \alpha$ is used to define the field of view, ( $f$ is the focal length of the collector). In the previous case, one could obtain a given resolution $\alpha$ in an $n \alpha$ field of view with considerable gain in exposure time. The principal advantage of the system is therefore (a) rejection of hard quanta and charged particles, (b) an improvement in signal-to-noise ratio, and (c) the ability to use small detectors in the focal plane which facilitates use of extremely thin windows.

\section{ONE DIMENSIONAL FOCUSING COLLECTOR}

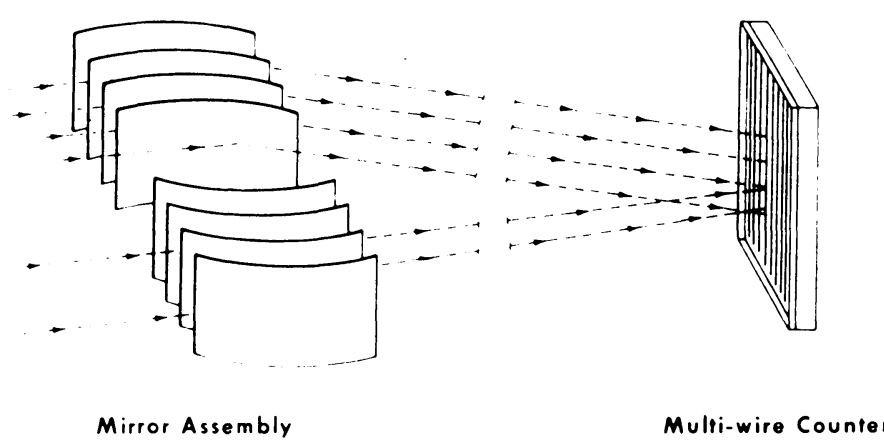

Fig. 4. A schematic representation of the operation of one-dimensional focussing collector used with a multi-wire proportional counter.

In all of these collectors the effective area is of the order of $100 \mathrm{~cm}^{2}$ at long wavelength. They differ, therefore, quite considerably from the very early versions flown by the ASE group on OSO 4 and by the London University group on OAO 2, which typically had collecting areas of a few square centimeters.

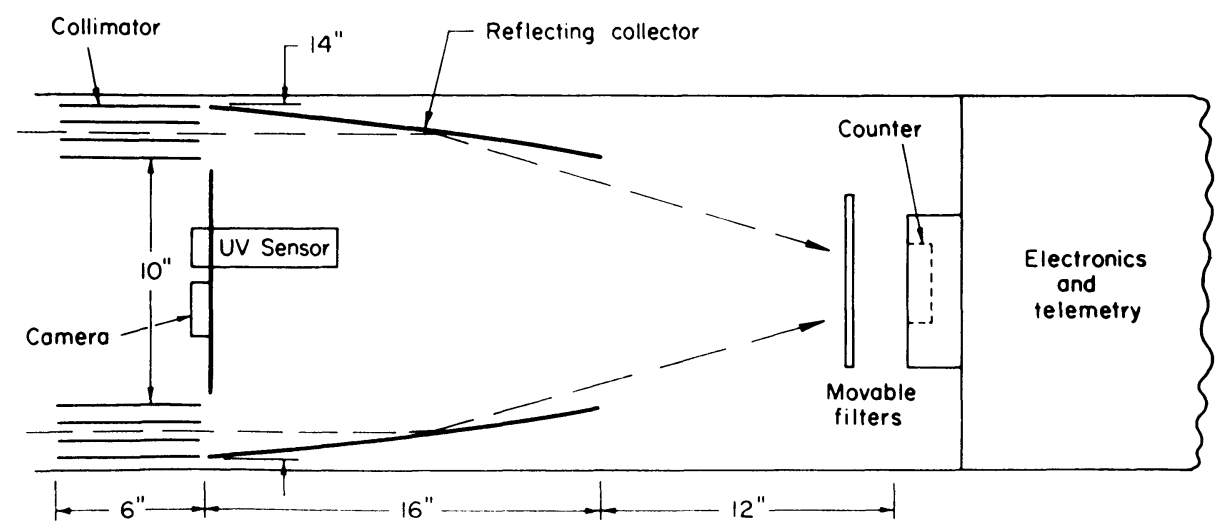

Fig. 5. Schematic representation of the Columbia rocket payload used for long wavelength surveys. (Courtesy R. Novick, Columbia University.) 


\section{Image-Forming Telescopes}

It was shown by Wolter (1952) that no single reflecting surface exists which results in a useful image at small angle of grazing incidence. For instance, the image resulting from an off-axis point source imaged by a single paraboloid with small grazing angle is approximately a circle having an angular radius equal to the distance off axis. The simplest truly focusing optics in the $\mathrm{X}$-ray region thus consists of two reflecting surfaces. Several simple configurations have been used in X-ray microscopy and more complex figured surfaces have been studied by Wolter. Recently interest for use of two reflection optics has been renewed by the desire to use imaging forming X-ray telescopes to study the structure of extended sources, such as the Crab Nebula and its Pulsar and $\mathrm{M} 87$.

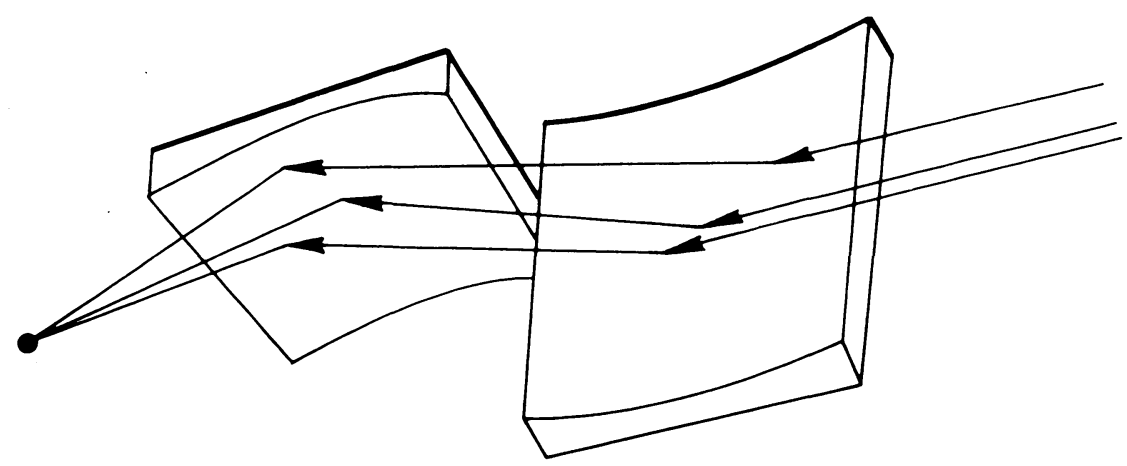

Fig. 6. Principle of operation of cross-mirror X-ray optics.

One of the simplest techniques which had been used in X-ray microscopy by Kirkpatrick and Baez as early as 1948 (Kirkpatrick and Baez, 1948) consists in the use of crossed mirror geometry. Figure 6 shows the principle of operation. Figure 7 shows a possible configuration with many surfaces which increase the collecting area of the device. This device, which is extremely simple to build and is capable of yielding large areas of collection with moderate angular resolution, has been investigated with some care by our group at AS\&E. We conclude that it is feasible to build very large scale versions of this device with resolutions in the focal plane as fine as a fraction of an arc minute. We have recently proposed this device for flight in one of the forthcoming NASA missions in collaboration with Columbia University and Cal Tech. The main advantage of the device is that it offers improved signal-to-noise ratio with respect to the one dimensional focusing device, that it permits the use of smaller detectors, and that it yields a true image of a source in the field of view.

Of more difficult realization are the two reflecting surfaces of telescopes which are based on the use of figures of revolution of conics. Hyperboloid and paraboloid configurations have been used by our group (Giacconi et al., 1965; Vaiana et al., 1968) for several years in solar studies as well as by Goddard Space Flight Center (Underwood and Muney, 1967). The principle of operation has been described extensively in 

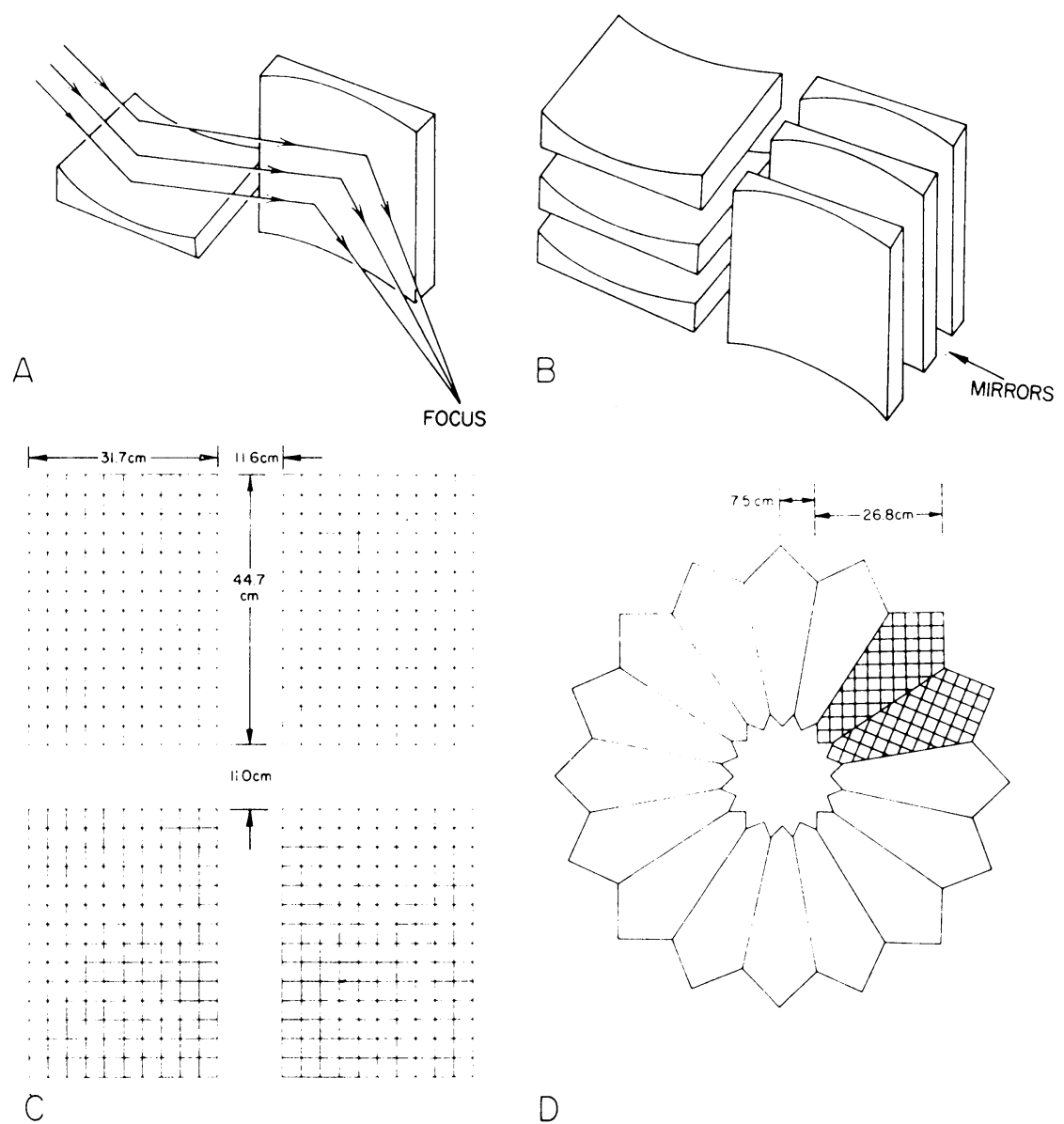

Fig. 7. Some practical realization of a multi-surface collecting mirror assembly. (A) shows the principle of operation; (B) the assembly of several surfaces; (C) the cross-section of a possible large mirror, and (D) more advanced packaging of the reflecting surfaces to obtain the largest possible collecting area within an envelope.

the literature (Giacconi et al., 1969) and is shown schematically in Figure 8. Figure 9 shows the improvement in resolution over the last few years in high angular resolution $\mathrm{X}$-ray observations using this type of telescope, which has resulted in significant results for solar physics. A rocket payload for stellar observations has been constructed by our group at AS\&E and is shown schematically in Figure 10. A 9.5 in. diameter grazing incidence telescope is used to focus $\mathrm{X}$-rays from 0.7 to $2 \mathrm{keV}$ on an image converter viewed by a photographic camera. The low efficiency of the telescope system and the short observing time will not permit the collection of sufficient statistics to utilize the intrinsic resolution of the optics. However, an observation of the Crab to be attempted this Fall might yield an angular resolution of about 20 arc sec sufficient to distinguish the emission from the Pulsar and the diffused nebula. A picture of the payload is shown in Figure 11. 

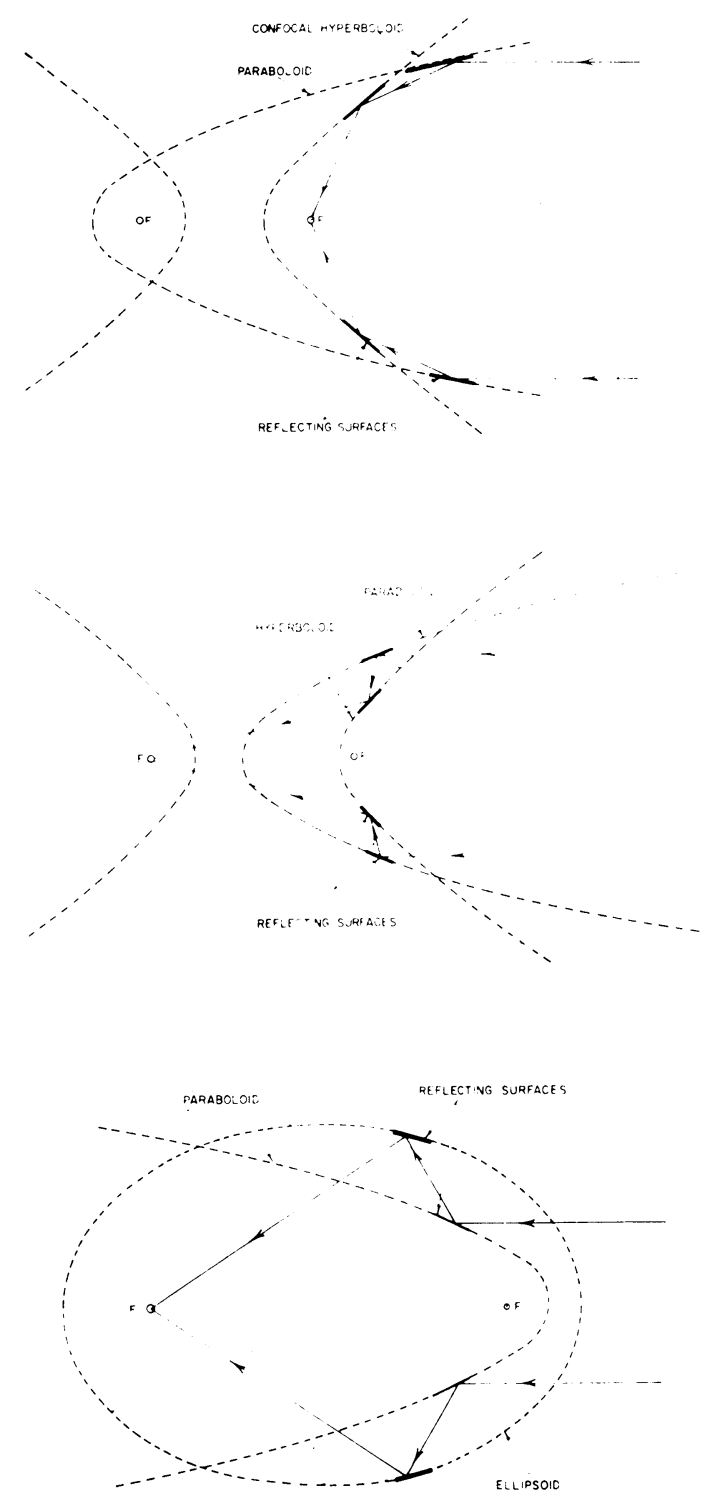

Fig. 8. Principle of operation of image forming X-ray telescopes. The three possible configurations above were the ones suggested by Wolter for X-ray microscopy.

\section{Detectors}

The major detector advance which has occurred in the past few years has been in the use of background rejection techniques for proportional counters. Two different approaches to this problem have been developed. The first is based on the use of multiwire proportional counters: an event which results in a pulse in only one wire 


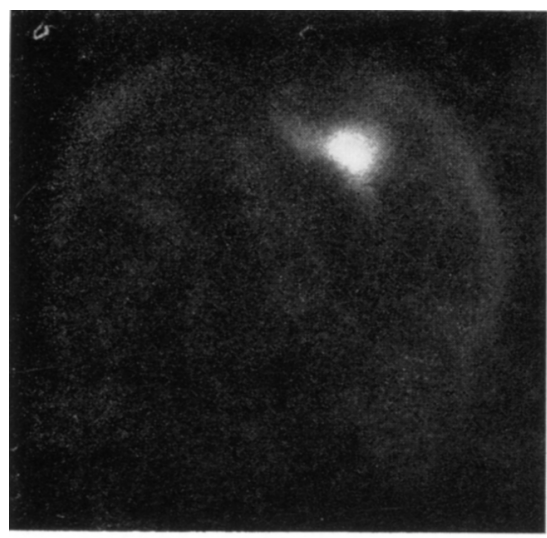

15 MARCH 1965

( AS\&E / GSFC)

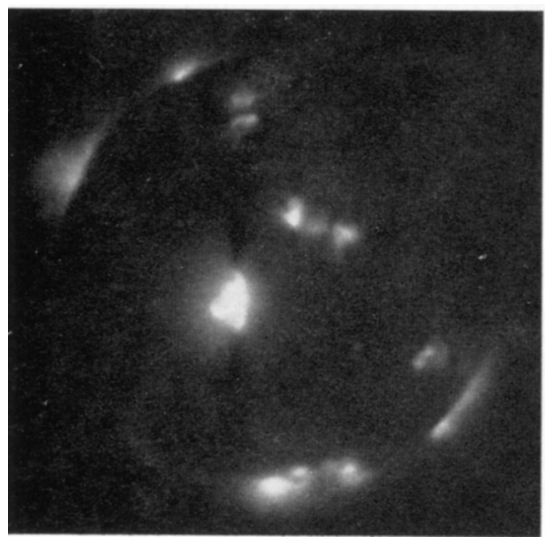

8 JUNE 1968

( AS\&E)

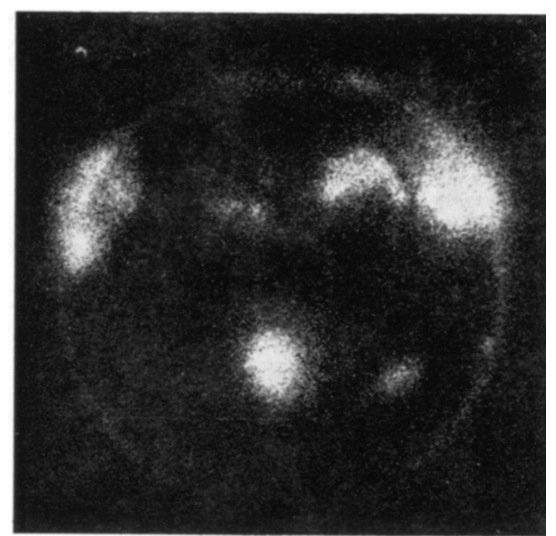

20 MAY 1966

( GSFC)

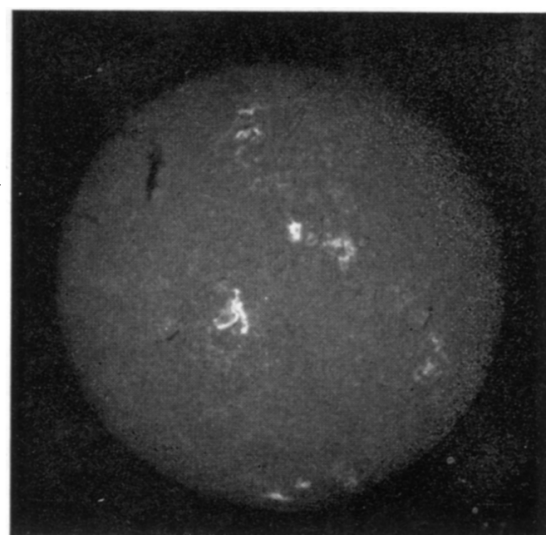

Ha

8 JUNE 1968

( COURTESY OF ESSA)

Fig. 9. Photographs of the sun obtained with grazing incidence X-ray telescopes over the past few years are shown in parts $\mathrm{A}, \mathrm{B}$ and $\mathrm{C}$ of the picture to illustrate the development of $\mathrm{X}$-ray optics during this period. An $\mathrm{H} \alpha$ photograph is also shown to illustrate the correspondence between $\mathrm{X}$-ray and $\mathrm{H} a$ features.

is accepted while events which also give rise to pulses in one or more adjacent wires are rejected. This method is, of course, based on the very small path length of the photoelectrons ejected in the gas by X-rays as compared to higher energy electrons. The method is quite effective over a wide range of energy (Cornell et al.. 1971; Kitamura et al., 1969). Somewhat simpler with respect to counter construction. but more 
complex with respect to electronic logic and applicable over a smaller range of wavelength is the pulse shape discrimination technique. This technique, first developed by a group at Leicester (Mathieson and Sanford, 1963), has been perfected and extensively utilized by our group and others (Giacconi et al., 1968). The method is also based on the small mean free path of X-ray ejected photoelectrons in the gas. This point-like energy deposition results in a fast rise time of the pulse in the anode wire

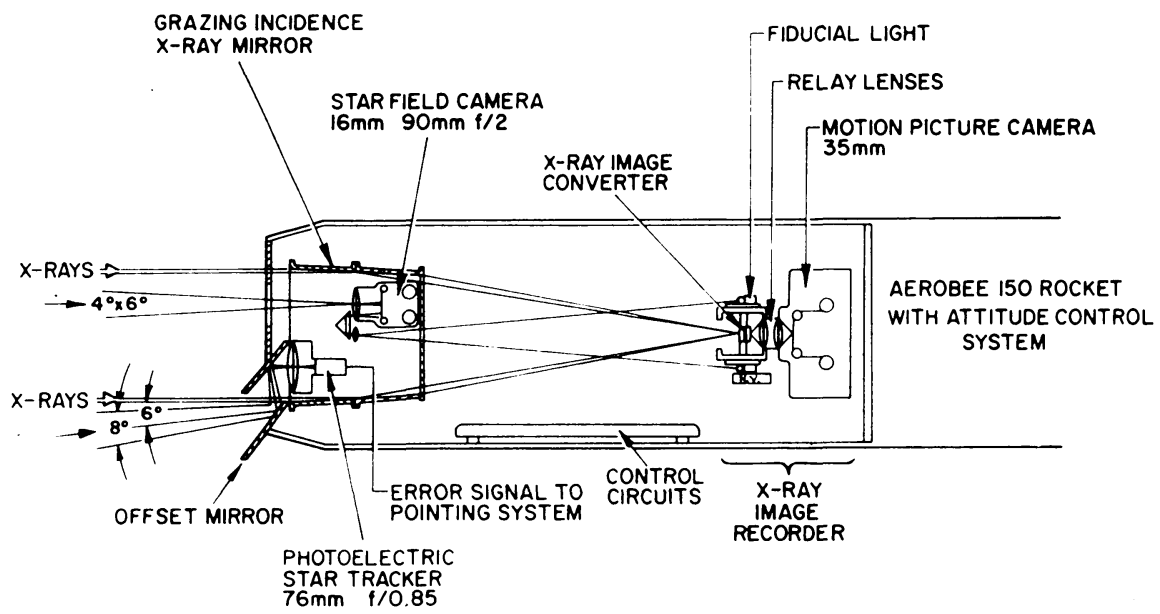

Fig. 10. Schematic representation of the AS\&E X-ray telescope experiment to be flown in an Aerobee 150 rocket. Note in particular the use of fiducial lights and corner prism reflectors to obtain an absolute in-flight calibration of the X-ray and visible light system alignment.

compared to the ones given by gamma rays or energetic charged particles. A convincing illustration of this technique at work is shown in Figure 12. Similar results have been obtained by other groups with either of the two techniques described above. This has yielded a factor 10 reduction in background which is equivelent to an increase of 10 in sensitive area or time of detection and has permitted a new round of high sensitivity rocket surveys which is just now coming to a close at the short wavelengths $1-10 \AA$.

Although not altogether new, it is remarkable that many groups have used extensively very thin window flow proportional counters in the last few years. Three examples of window transmission for detectors flown in the last year are shown in Figure 13. This trend reflects the desire to extend the surveys of background X-ray radiation and of individual sources to wavelengths as large as allowed by the transmission of interstellar gas.

\section{Imaging Detectors}

Multiwire counters have been used for the purpose of obtaining resolution in one dimension in connection with one dimensional focusing devices. P. Gorenstein will describe the use of a device of this type in a recent rocket flight. 
In connection with the use of simple, low angular resolution (1-10 arc min), but high effective area, two-dimensional imaging the possible use of imaging proportional counters has been investigated. Figure 14 shows the principle of use of a counter with many parallel wires to obtain information in the other dimension. Each anode wire is
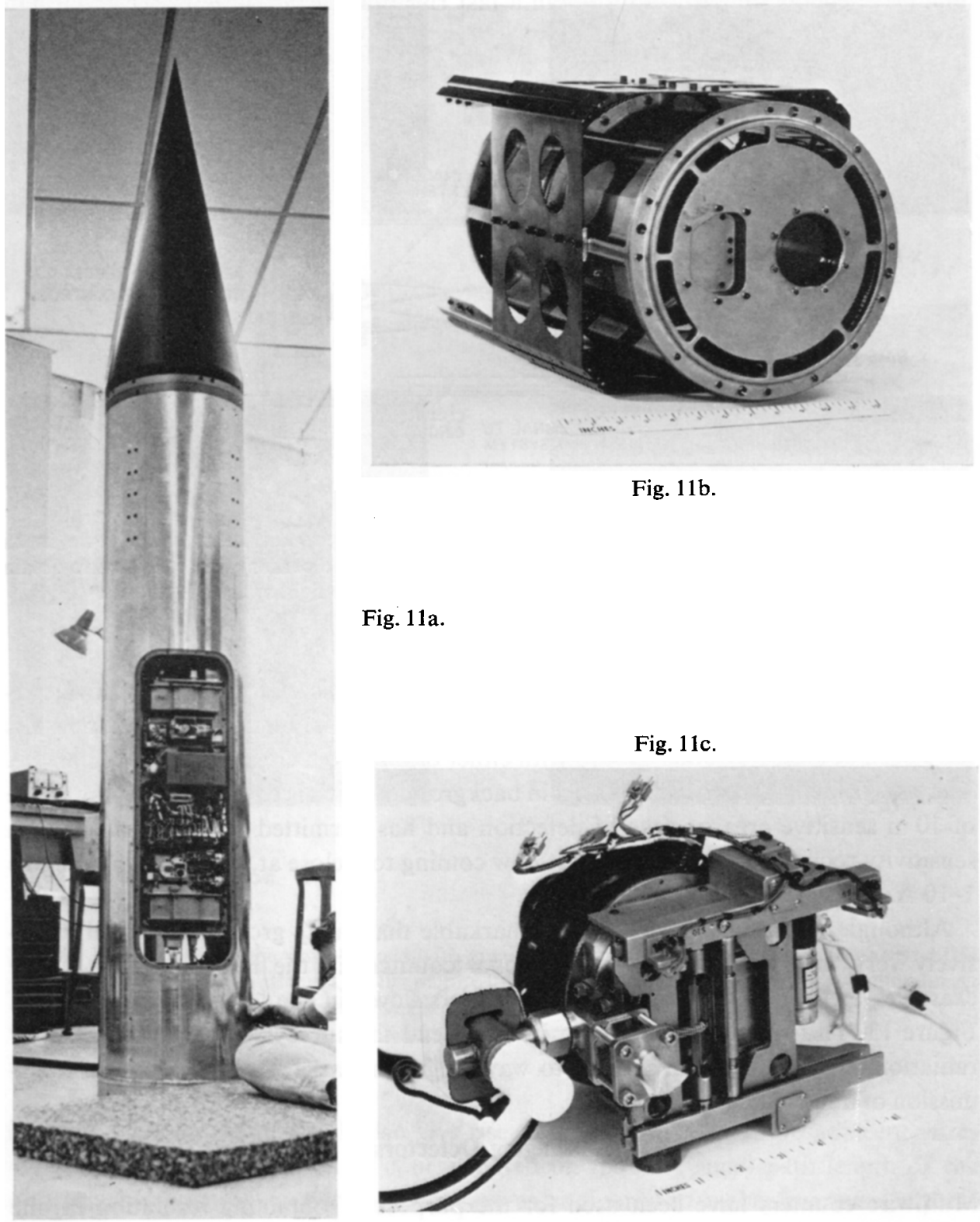

Fig. 11b.

Fig. 11a.

Fig. 11c.

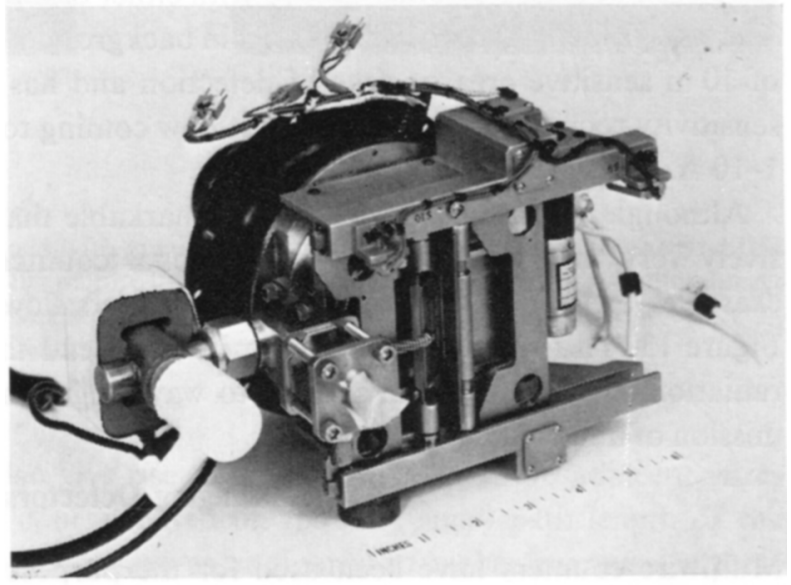

Fig. 11. Photograph of the ASE rocket payload including the high resolution $X$-ray telescope and image intensifier $\mathrm{X}$-ray detector. 
connected to a low input impedance charge sensitive preamplifier at each end. Because the anode resistance is large compared to the preamplifier input impedance, the division of the charge between the two preamplifiers can be used to sense this information.

In Figure 14 some typical results are shown. The number of counts vs. wire position as determined by $Q 1 / Q_{2}$ ratio is plotted for three different positions of a radioactive source. The technique is of quite enough precision to be used in two dimensional low resolution telescopes.

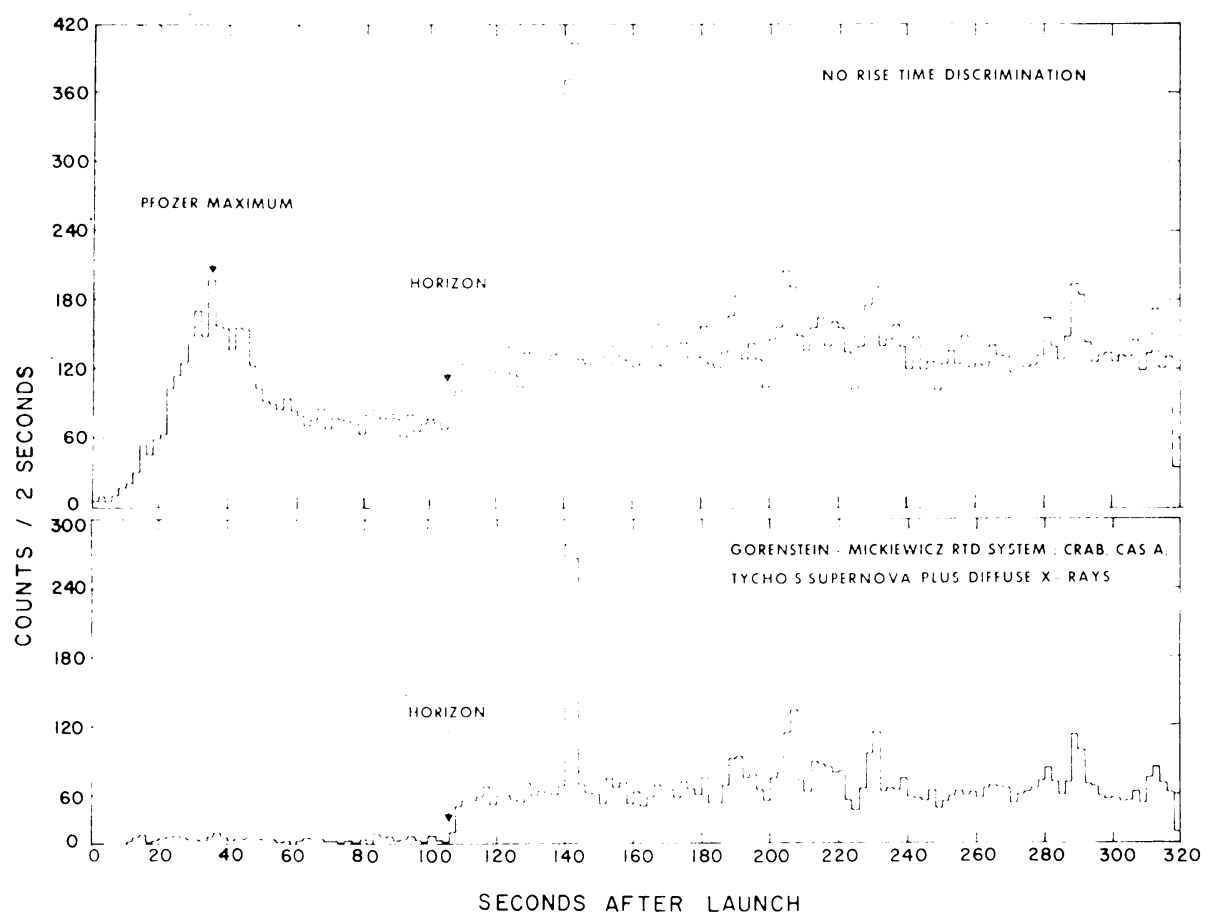

Fig. 12. Effect of rise time discrimination. The diagram above shows the observed counting rate in a proportional counter without rise time discrimination. The graph below shows the removal of background counts obtained by use of the technique. Note, in particular, the complete disappearance of the background effect due to cosmic rays at the Pfozer maximum and the relative slight decrease in sensitivity to $X$ rays.

Much higher angular resolution can be obtained by use of X-ray multichannel image converters. Their use has been described in the literature (Goodrich and Wiley, 1962) the principle of operation is shown in Figure 15. The advances in the field in the last few years are in the practical realization of X-ray detection systems incorporating this device and suitable for flight in rockets and satellites. The specific device to be used in the ASE rocket flight on Crab, mentioned above, is illustrated in Figure 16. A significant improvement of the intrinsic noise from these arrays has also been realized. Recent measurements in our laboratory show a noise rate of about $2 \times 10^{-7}$ 
events/channel s. The life expectancy of these devices has been studied by several groups (Egidi et al., 1969; Wolber et al., 1969) and shown to be primarily a function of total charge rather than hours of use. It can be estimated that with the expected fluxes from X-ray sources the life expectancy of an image intensifier would greatly exceed that of the spacecraft. The efficiency of the device has been measured by various groups and depends primarily on the photocathode substances used. Figure 17 shows a typical efficiency measurement obtained by Smith and Pounds, 1969, for $\mathrm{Mg} \mathrm{F}_{2}$.

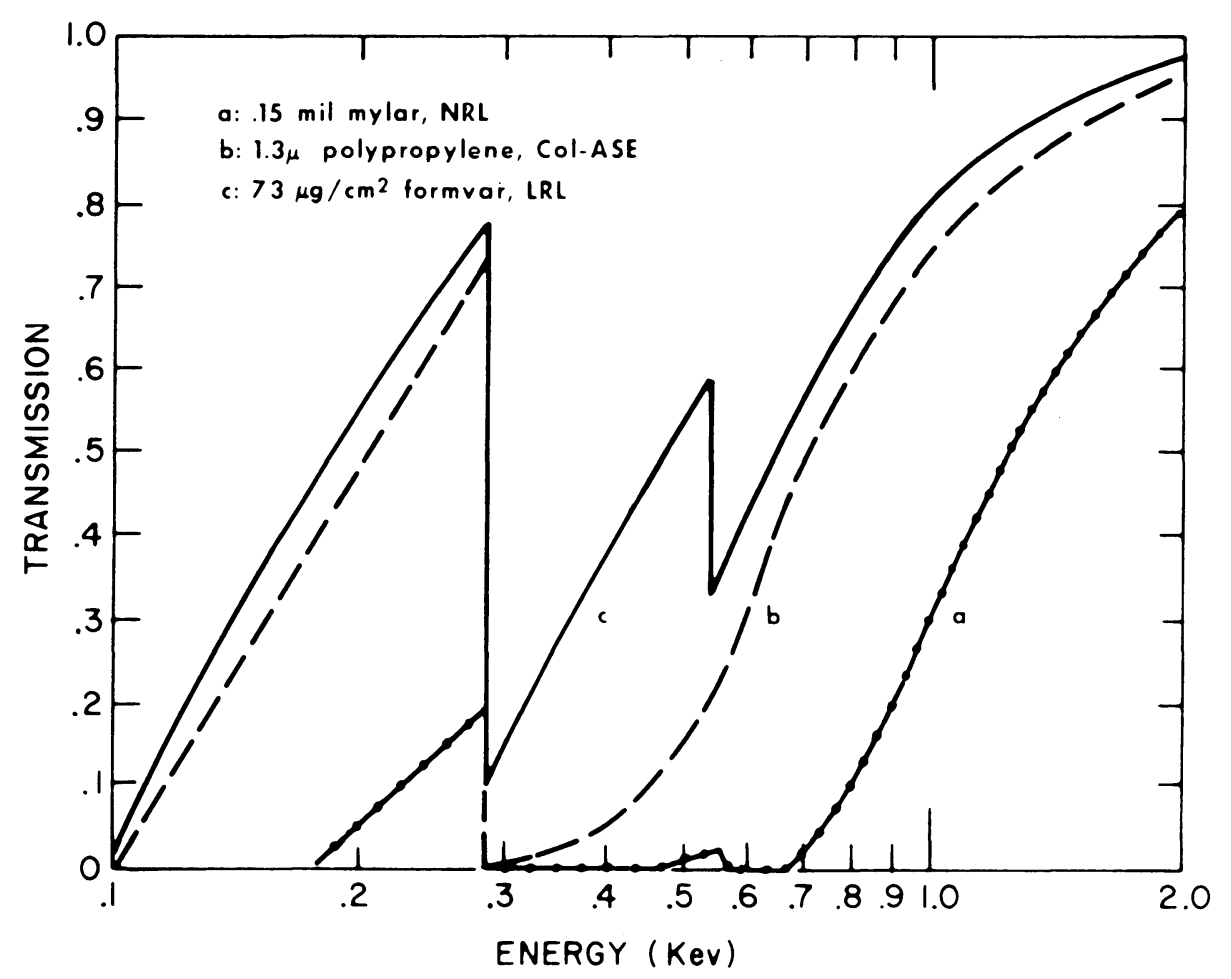

Fig. 13. Typical transmission characteristics of commonly used thin windows for proportional counters.

Film and television pick-up tubes have been used to detect the converted visible light image. Of particular importance for spacecraft use where the retrieval of photographic film is difficult is, of course, the use of TV systems. We have used SEC vidicons in our laboratory to detect the light flashes corresponding to single X-ray quanta with quite satisfactory signal-to-noise ratio as shown in Figure 18.

\section{Non-Dispersive Spectrometers}

Spectra obtained by pulse height analysis of proportional counters at low energy and scintillation counters at high energy remain the main tool of non-dispersive spec- 
troscopy in X-ray astronomy. Different mixtures of gases and special wire geometry have been used in most laboratories to improve the energy response of the gas counters. A typical spectrum obtained in a multiwire chamber by $\mathrm{P}$. Gorenstein is shown in Figure 19. The technique is essentially limited by ion statistic.

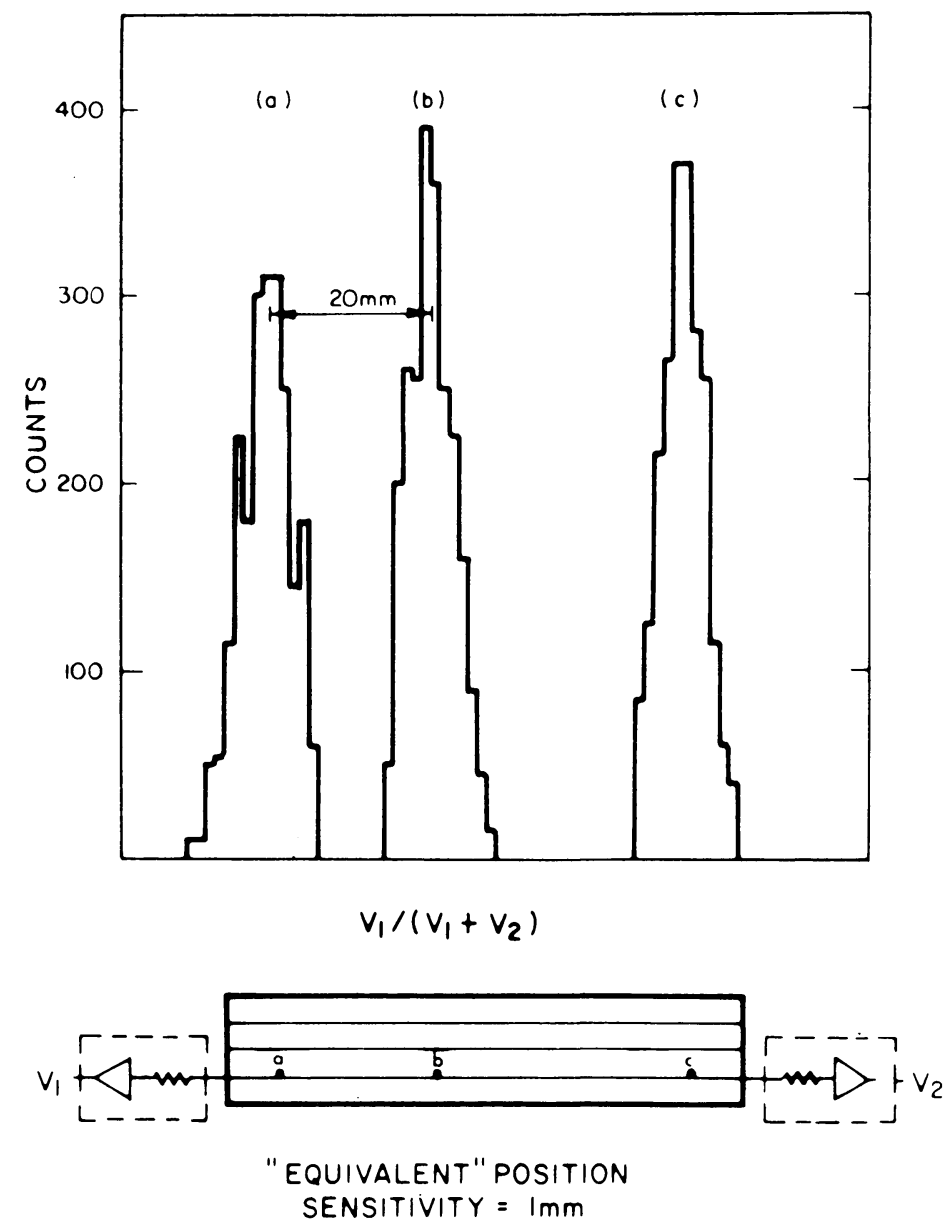

Fig. 14. The charged division technique in locating an event along a wire proportional counter is shown. The three peaks were obtained when the source was placed at positions A, B and C on the counter. The width of the distribution of the computed source position gives an indication of the precision of the technique.

Solid state detectors of the $\mathrm{Si}(\mathrm{Li})$ type appear to offer some promise of improvement particularly at the higher energies. Because of the small signals and lack of internal amplification the electronic noise must be kept to an absolute minimum. Therefore, $\mathrm{Si}(\mathrm{Li})$ detectors operate at low temperatures $(125 \mathrm{~K})$ and with low leakage currents $\left(<10^{-9} \mathrm{~A}\right)$. Practical devices appear at present to be limited in size to about $1 \mathrm{~cm}^{2}$ 
and in resolution to about $200 \mathrm{ev}$ FWHM at $1 \mathrm{keV}$. Their main advantage is the improved efficiency with respect to proportional counters and the fine resolution at high energy.

\section{Dispersive Spectroscopy}

Higher spectral resolution can be obtained, particularly at long wavelength by use of a slitless grating spectrometer. This instrument consists of a transmission grating in the ray path immediately behind an image-forming X-ray telescope. This technique.

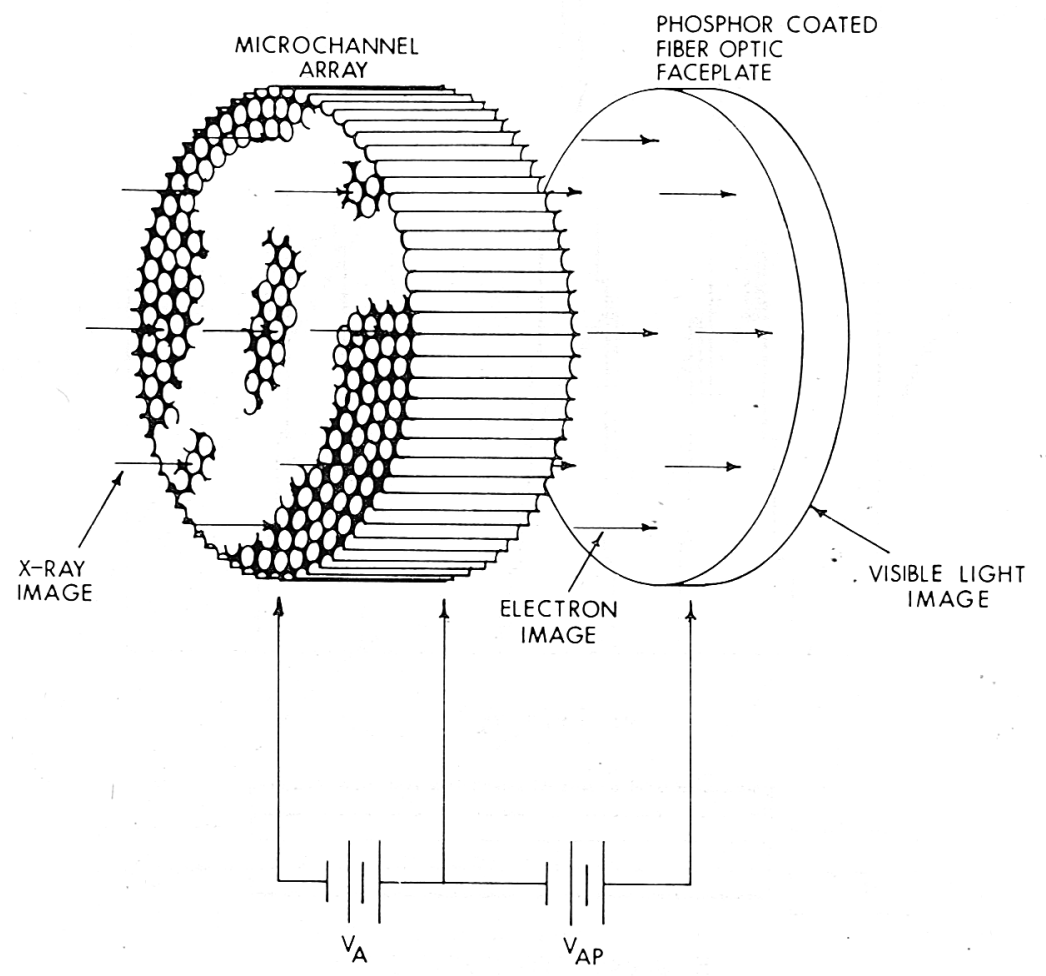

Fig. 15. X-ray image intensifier. The microchannel array consists of a bundle of small parallel tubes whose inner walls serve as photocathodes. A large gain results when the photoelectrons, accelerated axially down the tubes by an electric field, repeatedly strike the walls and generate secondary electrons.

first proposed by Gursky and Zehnpfennig, is described in detail in the literature (Giacconi et al., 1969). It can yield $\lambda / \Delta \lambda$ of about 50 at about $10 \AA$. Although used for solar studies, it has not yet found utilization for cosmic X-ray sources. In the future it might find use as a medium spectral resolution, but high sensitivity, technique. The high sensitivity is given by its ability to examine the entire spectral region of interest at once. Much higher spectral resolution of the order of $10^{3}$ to $10^{4}$ can be obtained using crystal spectroscopy. 


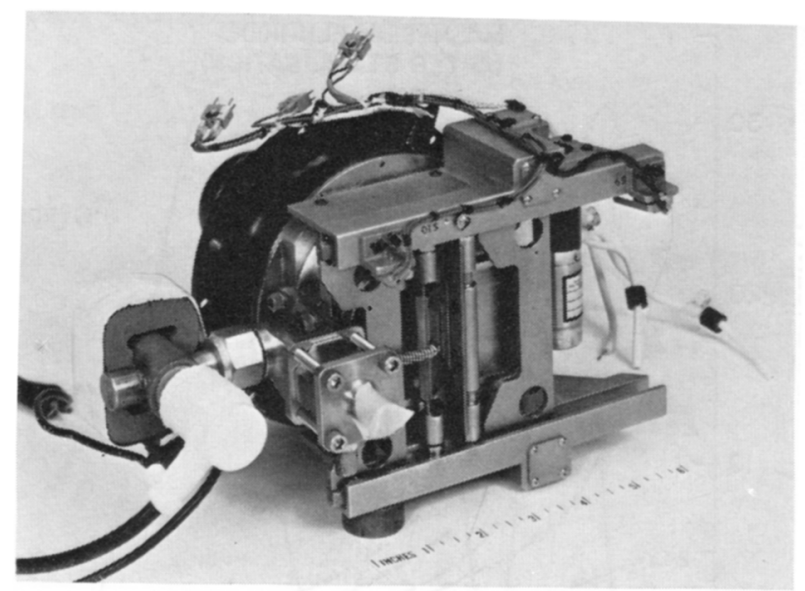

Fig. 16a.

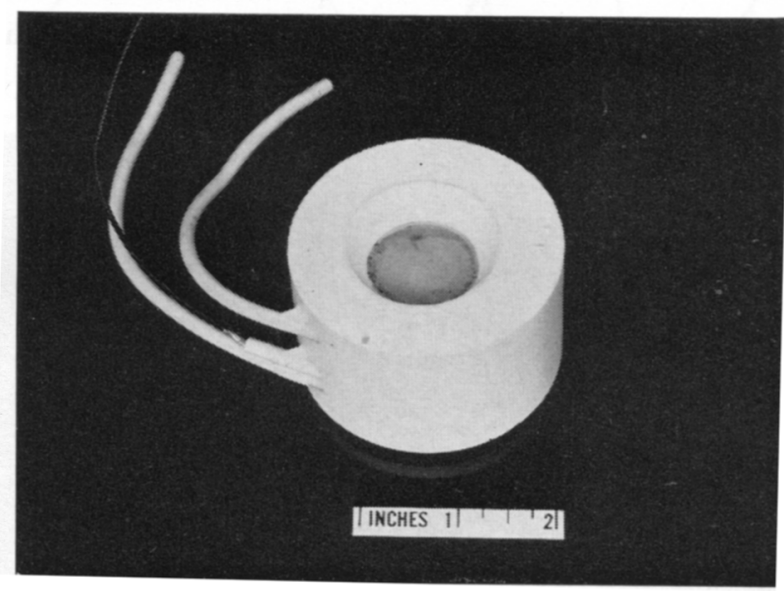

Fig. 16b.

Fig. 16. Photograph of the ASE image intensifier system for detection of soft X-ray images. Above is shown the vacuum chamber containing the image intensifier with the door closed. Below an image intensifier encapsulated for flight is shown removed from its housing.

Non-focusing plane crystal spectrometers have been used extensively in solar studies (Blake et al., 1965; Neupert et al., 1967; Evans and Pounds, 1968; Walker et al., 1967) and a typical spectrum is shown in Figure 20. The main difficulty of utilizing this technique for celestial X-ray sources is due essentially to the low intensity of the observed X-ray flux.

Very large arrays of crystals had to be developed to improve sensitivity and the integrated reflectivity of the crystal themselves maximized. This has been the direction of the efforts of the Columbia Astrophysics Laboratory in the last year. Angel and Weisskopf (1970) have shown that the most efficient use of Bragg reflection for spec- 


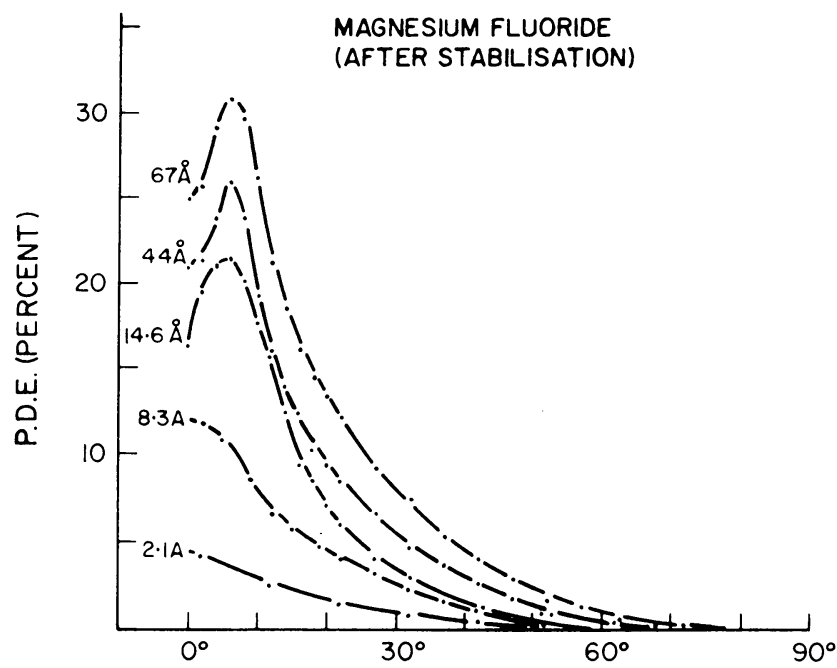

Fig. 17. Quantum efficiency of magnesium fluoride at different wavelength obtained by Smith and Pounds, 1968.

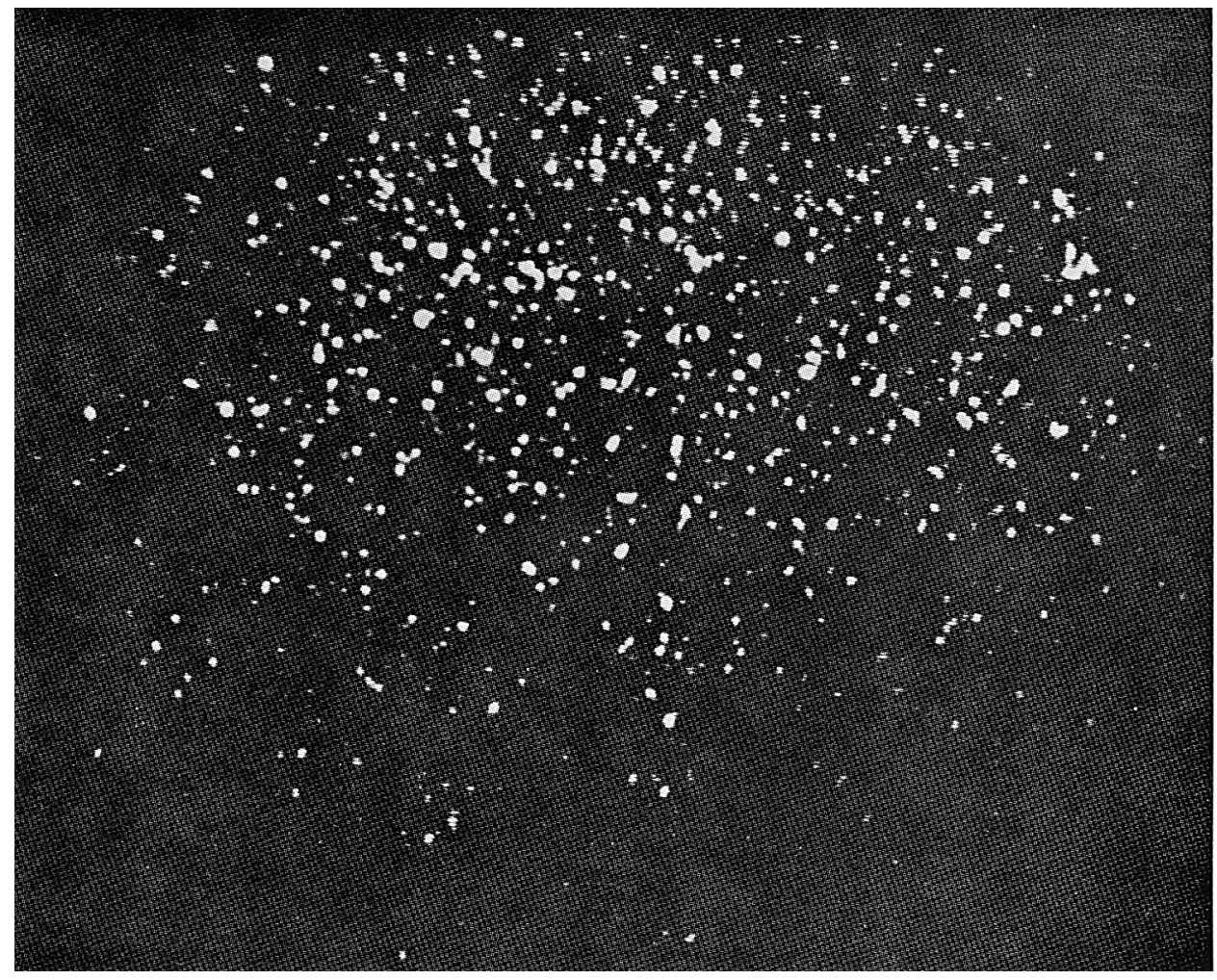

Fig. 18. T. V. monitor output resulting from an SEC camera and image intensifier illuminated with soft X-rays. The bright spots demonstrate the system sensitivity to individual X-ray photons. 


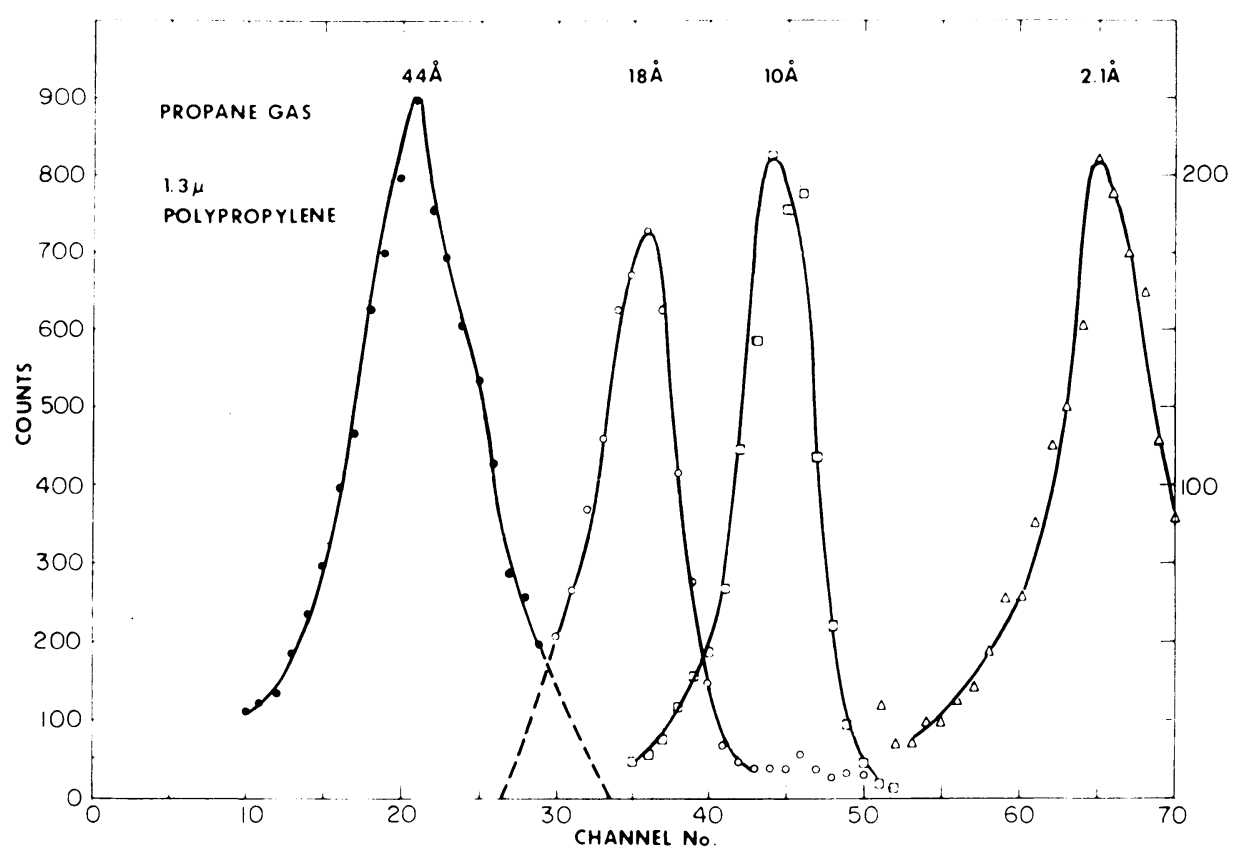

Fig. 19. Resolution of a thin window proportional counter to characteristic $\mathrm{X}$ radiation of different wavelength obtained at AS\&E with a multi-wire proportional counter.

troscopy is obtained by exploiting the properties of mosaic crystals with high integrated reflectivity, in particular, lithium hydride, graphite and tungsten disulphide. Mosaic crystals are such that perfect alignment of crystal planes is maintained only over microscopic domains so that a larger portion of the spectrum can be reflected at any angle of incidence that occurs in perfect crystals. This results in a higher integrated

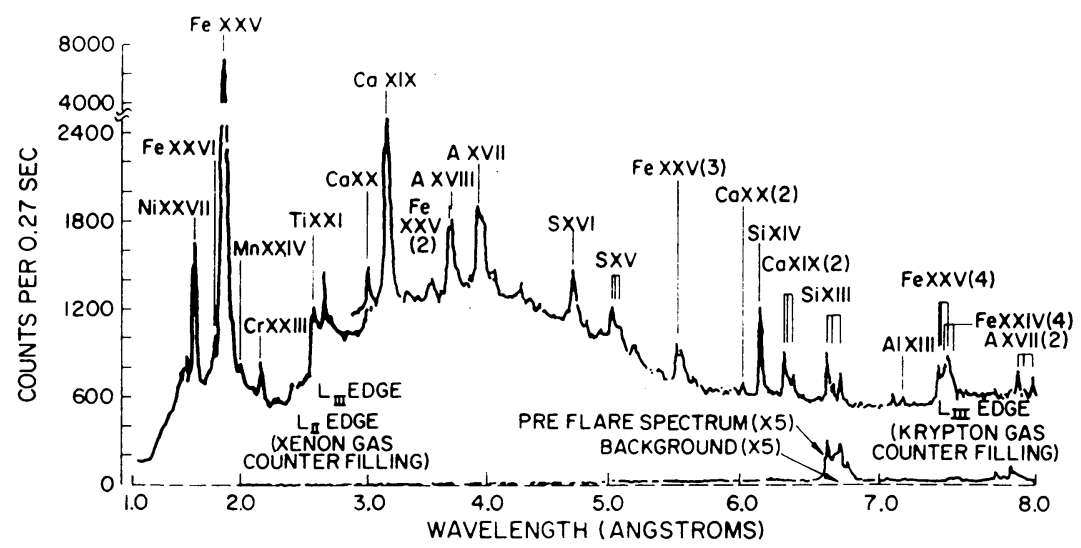

Fig. 20. Solar flare spectrum obtained from OSOV (Neupert, 1969). The calculated plasma temperature is approximately 27 million deg. Similar spectra would be expected from stellar sources having this temperature and density. 
reflectivity by factors of 10 to 100 at the expense of coarser resolution if the crystals are employed in the conventional manner. A rocket payload which utilizes the above approach, is shown schematically in Figure 21 ; the experiment will be described in detail by Prof. Novick at this meeting.

I believe that similar developments are being undertaken by the Leicester University group in England and perhaps K. Pounds will report on their techniques at this meeting.

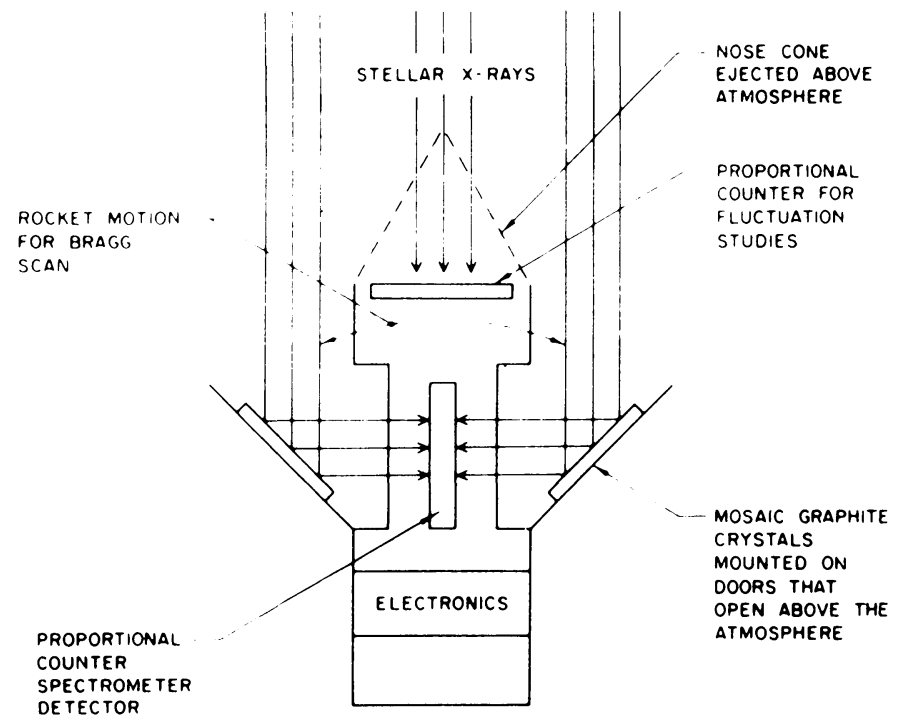

Fig. 21. Schematic diagram of a rocket payload for the measurement of X-ray polarization which is being prepared by the Columbia group (Courtesy R. Novick, Columbia).

The use of imaging X-ray telescopes permits the use of Bragg crystal spectrometers in a variety of configurations which permit the achievement of larger signal to noise ratios and finer resolutions. To continue with the work at Columbia, the same group has tested in the laboratory a configuration similar to the one illustrated schematically in Figure 22. When used in conjunction with mosaic crystal in the configuration illustrated, the intrinsic resolution of the X-ray telescope can be used to determine accurately the spectral distribution within the range of wavelengths reflected by the mosaic. Thus, although crystals with high integrated reflectivity show of necessity a broad rocking curve, they can be used in this fashion to obtain high resolution spectra (Angel and Weisskopf, 1970).

A different approach has been used by the MIT group. Schnopper and Kalata have studied the properties of curved crystal spectrometers to be used at the focus of imaging X-ray telescopes (Schnopper and Kalata, 1969a). They have developed a new technique of crystal mapping which allows the achievement of extremely high resolution of about $2 \times 10^{4}$ with high efficiency and without the requirement for high resolution image detection. The principle of the technique is shown in Figure 23 and a 


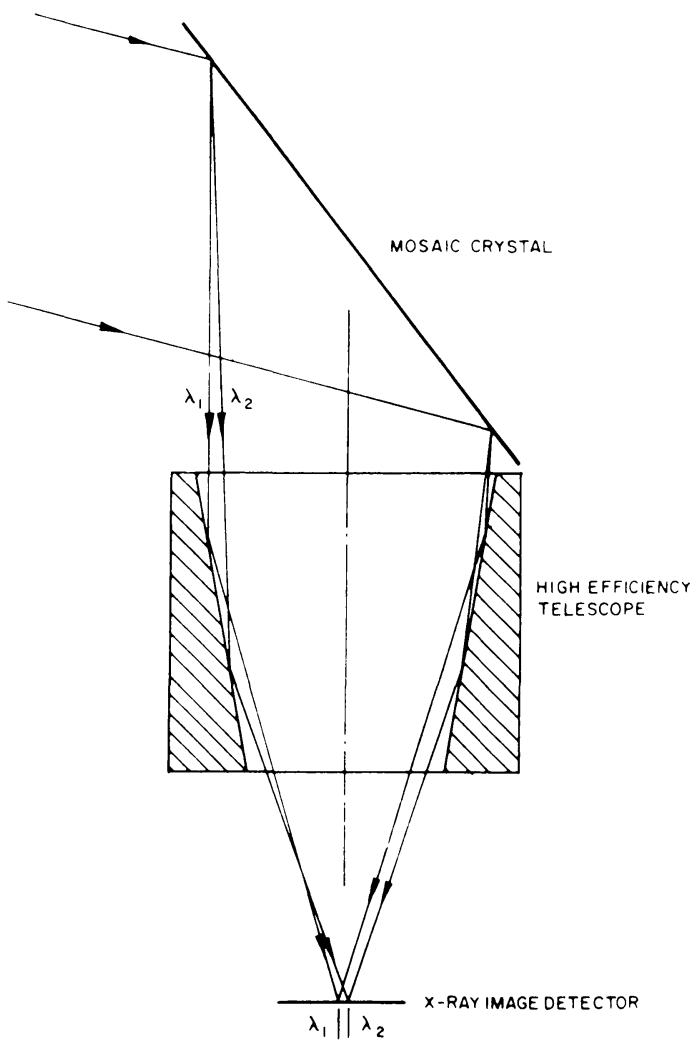

Fig. 22. Conceptual diagram of objective crystal spectrometer. The angle between the source direction and the reflected ray determines the wavelength of the radiation.

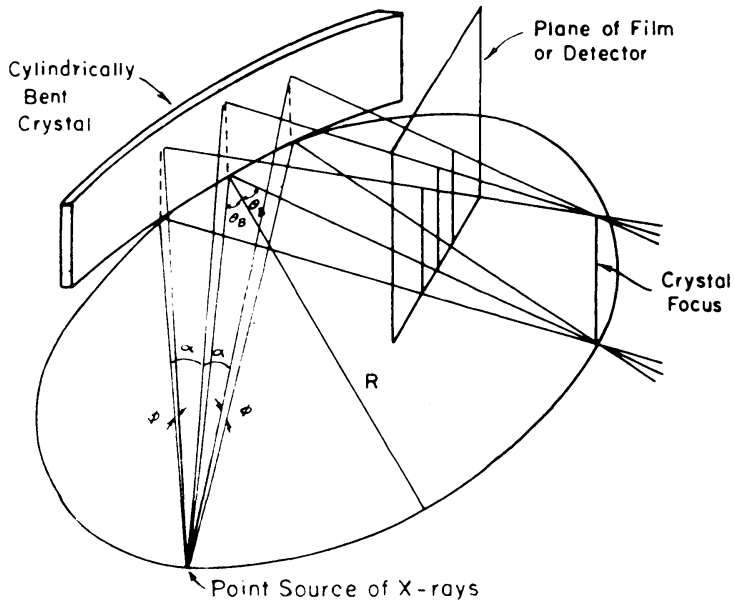

Fig. 23. Schematic diagram of an off-focus detection technique for an X-ray spectrometer as proposed by the MIT group. 


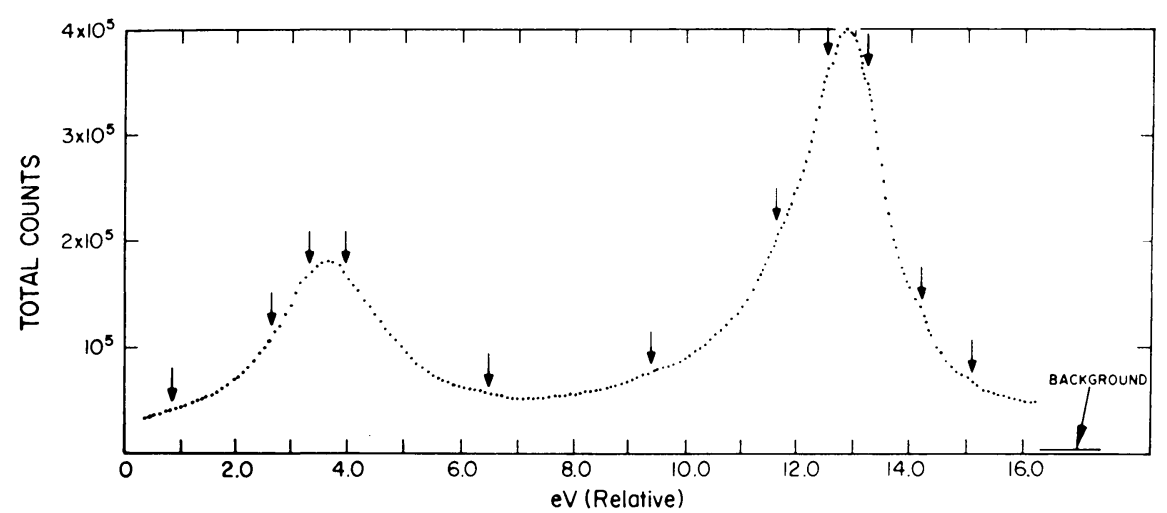

Fig. 24. A spectrum of the CRK $\imath_{1} \varkappa_{2}$ region obtained from a number of corrected verticalscans. The features which were observed are indicated by arrows. The X-ray tube was operated at $20 \mathrm{kV}$ and $20 \mathrm{~m} \AA$. (Schnopper and Kalata 1969.)

sample of data on the spectrum of $\operatorname{Cr} \mathrm{N} \alpha, \alpha_{2}$ obtained in the laboratory is shown in Figure 24. The use of this technique has been proposed to NASA for one of the forthcoming X-ray astronomy missions.

\section{Polarimeters}

The interest in stellar X-ray polarization is stimulated by the hope of obtaining information about the nature of emission processes. Thompson scattering polarimeters were utilized by the Columbia group in a search for X-ray polarization of Sco-X-1 (Angel et al., 1969) with negative results. The instrument was sensitive in the region above $5 \mathrm{keV}$ with a cross-sectional effective area of about $30 \mathrm{~cm}^{2}$. A limit of $10 \%$ was placed on the polarization of Sco X-1. New techniques utilizing Bragg reflection at $45^{\circ}$ with or without focusing optics have been proposed by Schnopper and Kalata (1969) and by Angel and Weisskopf (1970). These techniques offer an attractive way of detecting polarization at lower energies. Schnopper and Kalata suggested the use of a focusing curved crystal as shown in Figure 25 and Angel and Weisskopf have proposed the use of flat crystals at the focus of a grazing incidence lens as shown in Figure 26. The authors estimate that polarization of the order of 5 to $10 \%$ of Sco X-1 could be detected at longer wavelengths even from a short rocket flight.

\section{Satellite Systems}

After this brief description of progress in each of the elements of an X-ray astronomy payload, I would like to conclude giving two examples of satellite systems in which some of these techniques are utilized.

The first example is SAS A, a spacecraft in advanced state of completion and the first to be launched in the new series of satellite experiments which I mentioned above. SAS A (Giacconi et al., 1968a) will be the first satellite payload totally devoted to 
X-ray observations. The vital characteristics are given in Table II. Figure 27 shows the instrument section in the process of integration at the Applied Physics Laboratory of Johns Hopkins University.

To illustrate how we expect SAS A might increase our knowledge of the X-ray sky, we have prepared on the basis of theoretical expectation of galactic and extragalactic source numbers a random computer plot of the X-ray sky as it might appear at SAS A sensitivity. This is shown in Figure 28. While this pedagogical device is, of course, not a prediction, it vividly illustrates the difficulties we will encounter in

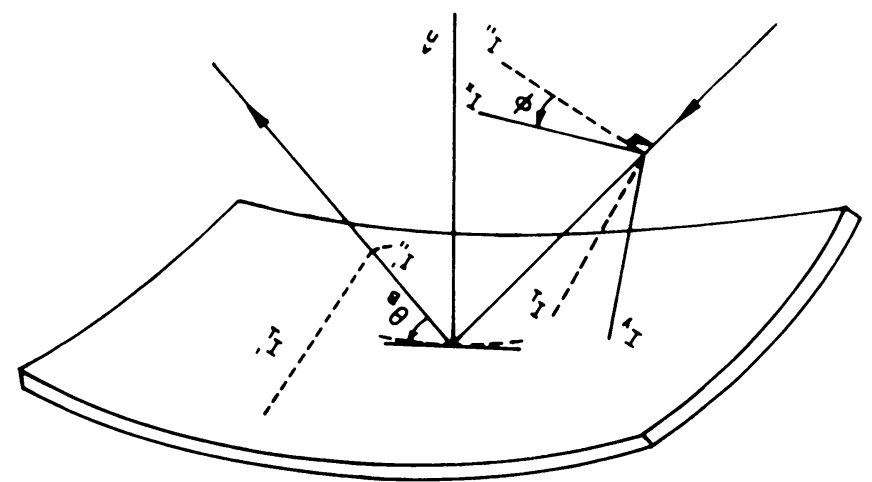

Fig. 25. Reflection of X-rays from a bent crystal with a Bragg angle near $45^{\circ}$ to study polarization of celestial X-ray sources. (Schnopper and Kalata, 1969.)

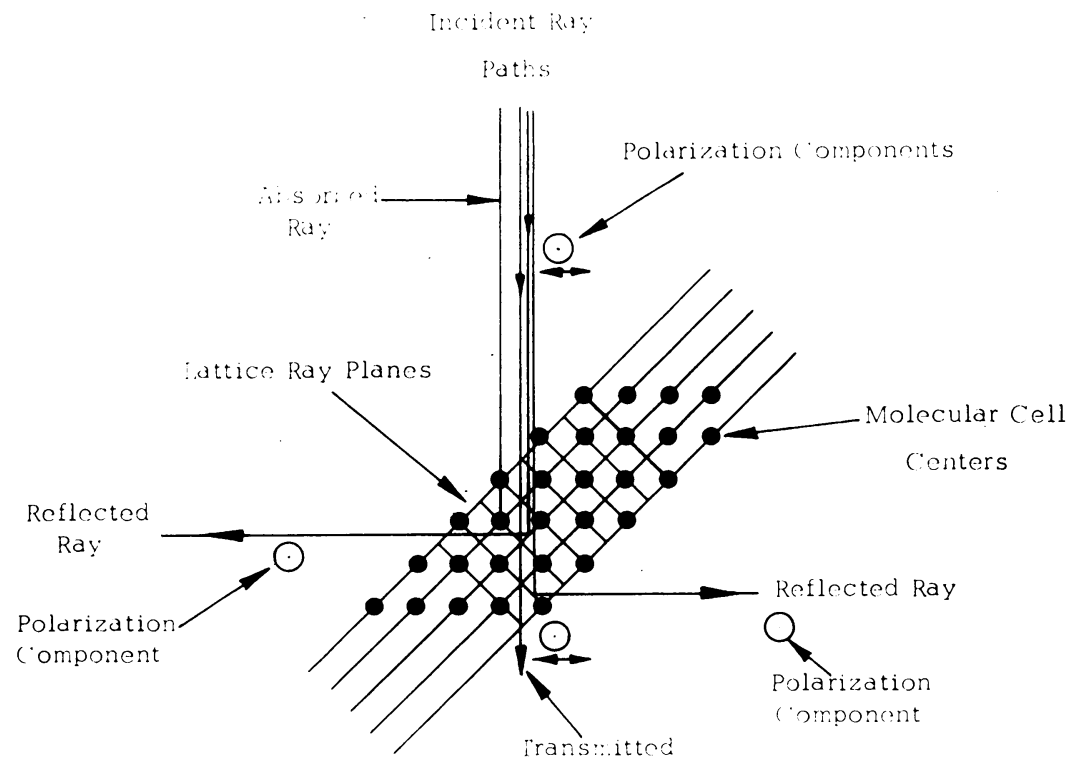

Fig. 26. Schematic representation of transmitted and reflected rays in a Bragg Crystal Polarimeter. Crystals of LiH have cubic symmetry and therefore reflect incidence radiation in two directions. 
utilization of more and more sensitive detectors unless they also are given sufficient angular resolution to permit us to avoid source confusion.

As the second example of a very advanced satellite system, I would like to mention a project on which I myself and several colleagues of different institutions have been

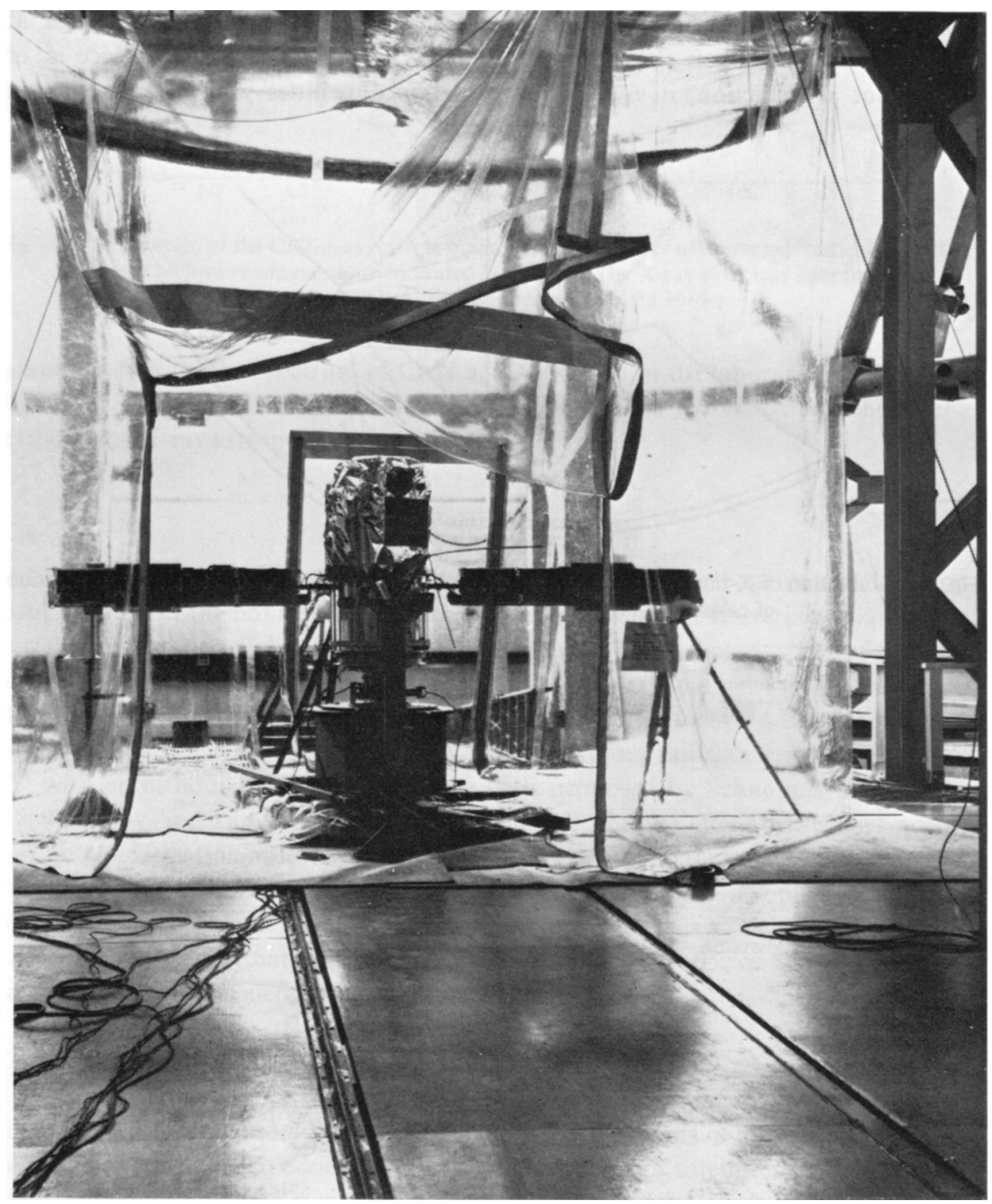

Fig. 27. The SAS A spacecraft in flight configuration during system testing. Note above the solar paddles, the X-ray detecting area (the black rectangle) and the star sensor. The aluminized mylar cover is used for thermal shielding. (NASA photograph: courtesy National Aeronautics and Space Administration). 


\section{TABLE II}

\section{SAS A}

Institution
Launch
Spacecraft SAS A
Instruments

Energy range

Min detectable source intensity Source Location

Source structure Spectral resolution Time resolution
AS\&E

1970

scanning, spin 0.1 repm

$140 \mathrm{lb}$

$800 \mathrm{~cm}^{2}$ proportional counters

FoV $0.5^{\circ} \times 5^{\circ}, 5^{\circ} \times 5$

$2-20 \mathrm{keV}$

$5 \times 10^{-5}$ Sco X-1

1 arc min for $10^{-3}$ Sco X-1

25 arc min for $5 \times 10^{-5}$ Sco X-1

$\sim 1^{\circ}$

$\lambda / \Delta i \simeq 10$

$0.1 \mathrm{~s}$

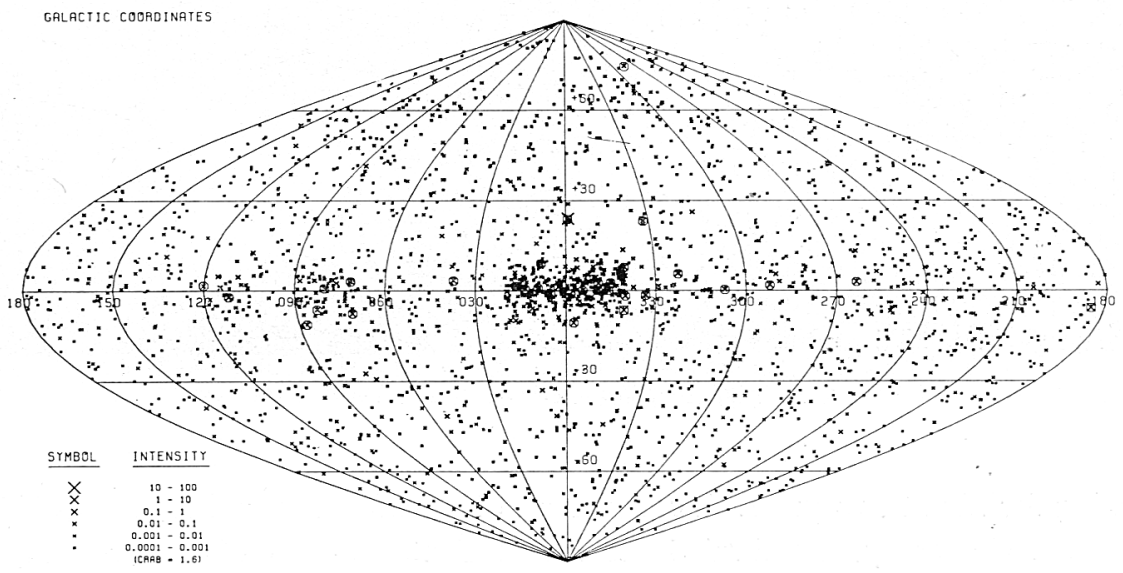

Fig. 28. A computer simulation of the X-ray sky as might be observed by the SAS experiment. Know sources have been plotted at their position. Reasonable guesses have been made with respect to the population, density and distribution for galactic and extragalactic sources. The computer program yielded a random distribution of these sources according to the prescription. This figure illustrates the complex nature of the X-ray sky and the possibility for confusion which may arise as a result of the much improved sensitivity of the forthcoming experiments.

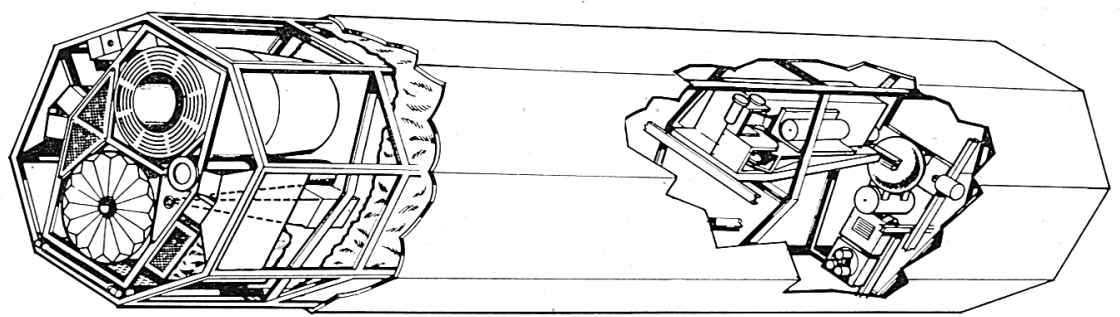

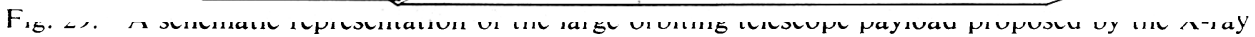
groups of AS\&E, Columbia University, Goddard Space Flight Center, and MIT, for inclusion in the forthcoming High Energy Observatory NASA mission. 


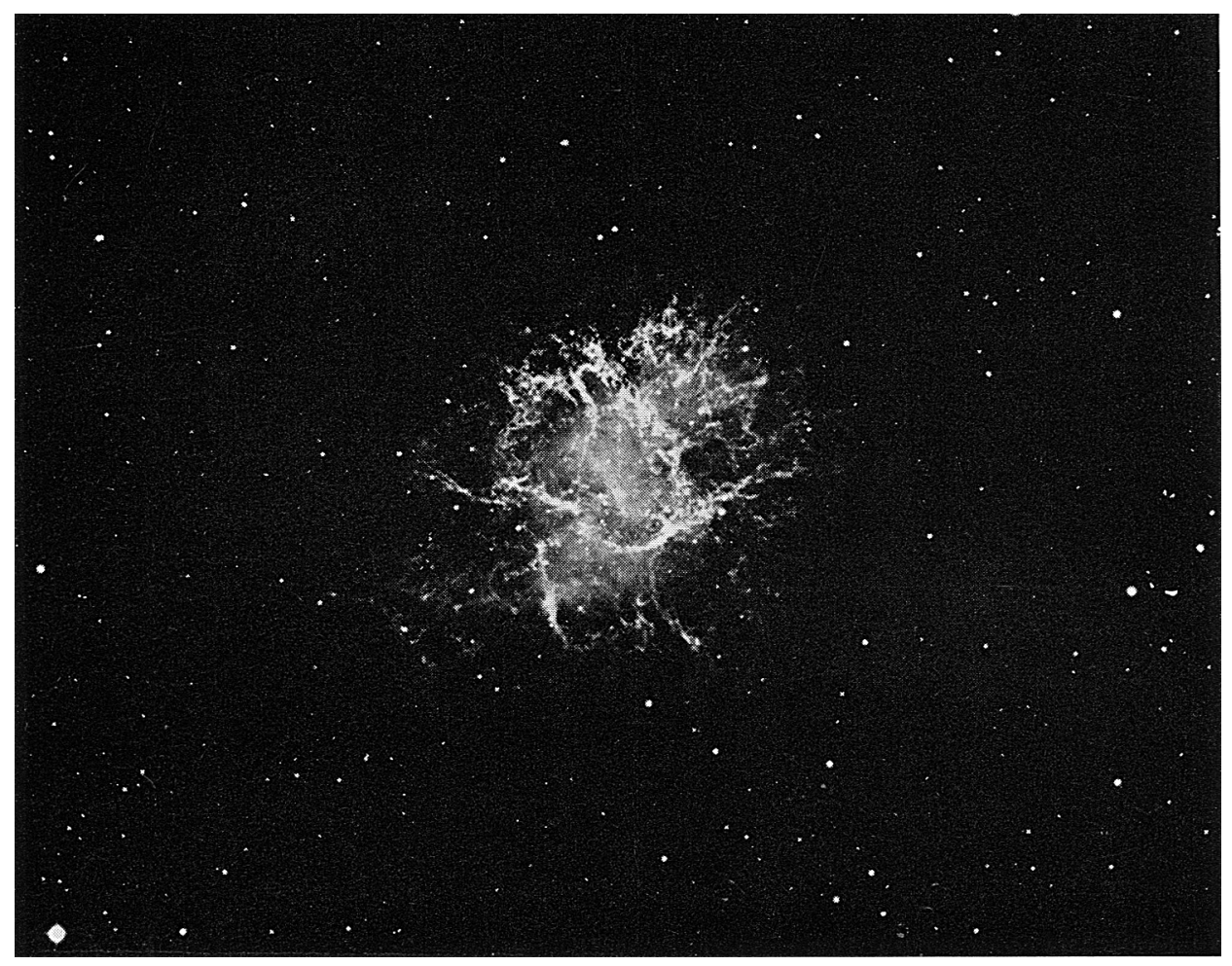

Fig. 30a.

working for about two years. A consortium of scientists at four institutions (AS\&E, Columbia University, Goddard Space Flight Center, and MIT) has recently proposed a large orbiting X-ray telescope (LOXT) to NASA for inclusion in the High Energy Astronomical Observatory presently in the planning phase (X-ray Astronomy Groups, 1969). This experiment (shown in Figure 29) which might be flown in the middle to late 1970's, embodies almost all the technical advances described above. The vital statistics are shown in Table III. To illustrate the capabilities of this instrument we have prepared Figure 30 which shows the Crab Nebula, the large Magellanic Clouds, and M 31. Listed in the side column are the X-ray, visible and radio characteristics of Crab and Pulsar NP0532 and of the same objects if removed at the distance of two extragalactic objects. Crab could be detected and its structure resolved by LOXT at the distance of the LMC and it could be detected and localized even at the distance of M 31. The pulsar NP 0532 could also be detected as far as M 31 as an individual source although its visual magnitude would by then have reached $M_{v}=+30$. Thus, we can expect in the coming decade qualitative improvements in our understanding of processes giving rise to the observed high energy photons in galactic and extragalactic objects. X-ray telescopes will play an increasingly important role in this very exciting new phase of X-ray astronomy. 


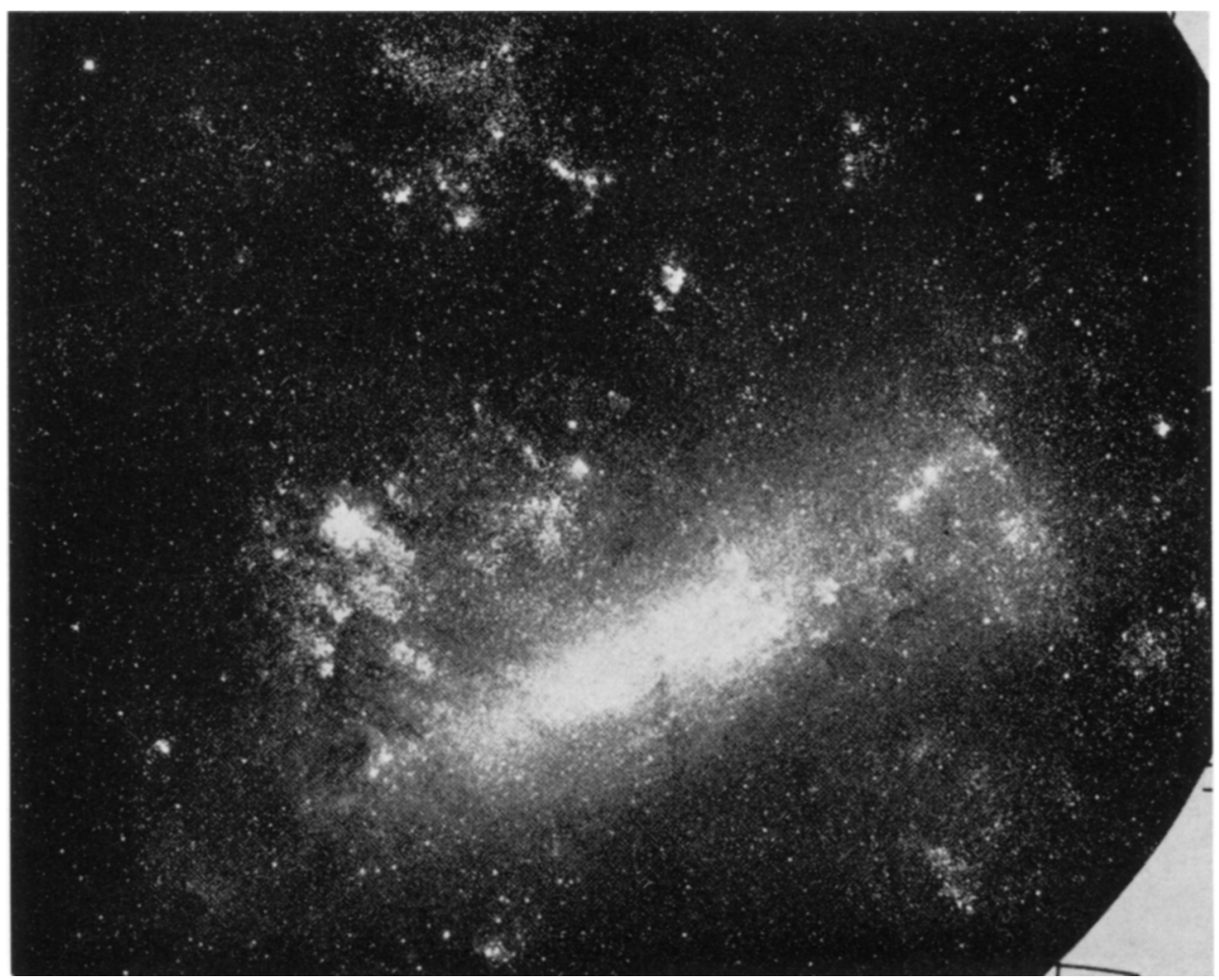

Fig. 30b.

TABLE III

LOXT

Institution

Spacecraft

Launch

Instruments

\section{Energy range}

Minimum detectable source intensity

Source location

Source structure

Spectral resolution

Time resolution

Polarization
AS\&E, CU, GSFC, MIT

HEAO - pointing 1 arc min

1975-1978

$12000 \mathrm{lb}$.

1 meter diameter X-ray telescopes

Image detectors, spectrometers,

polarimeters

0.2 to $7 \mathrm{keV}$

$10^{-7}$ Crab Nebula in A Day

$2 \times 10^{14} \mathrm{erg} / \mathrm{cm}^{2} \mathrm{~s}$ in $1000 \mathrm{~s}$

$1 \mathrm{arcsec}$

5 arc sec

$\lambda / \Delta \lambda \simeq 10^{4}$

$0.1 \mathrm{~ms}$

$1{ }^{\circ}$ in Crab Nebula in $1 \mathrm{~d}$ 


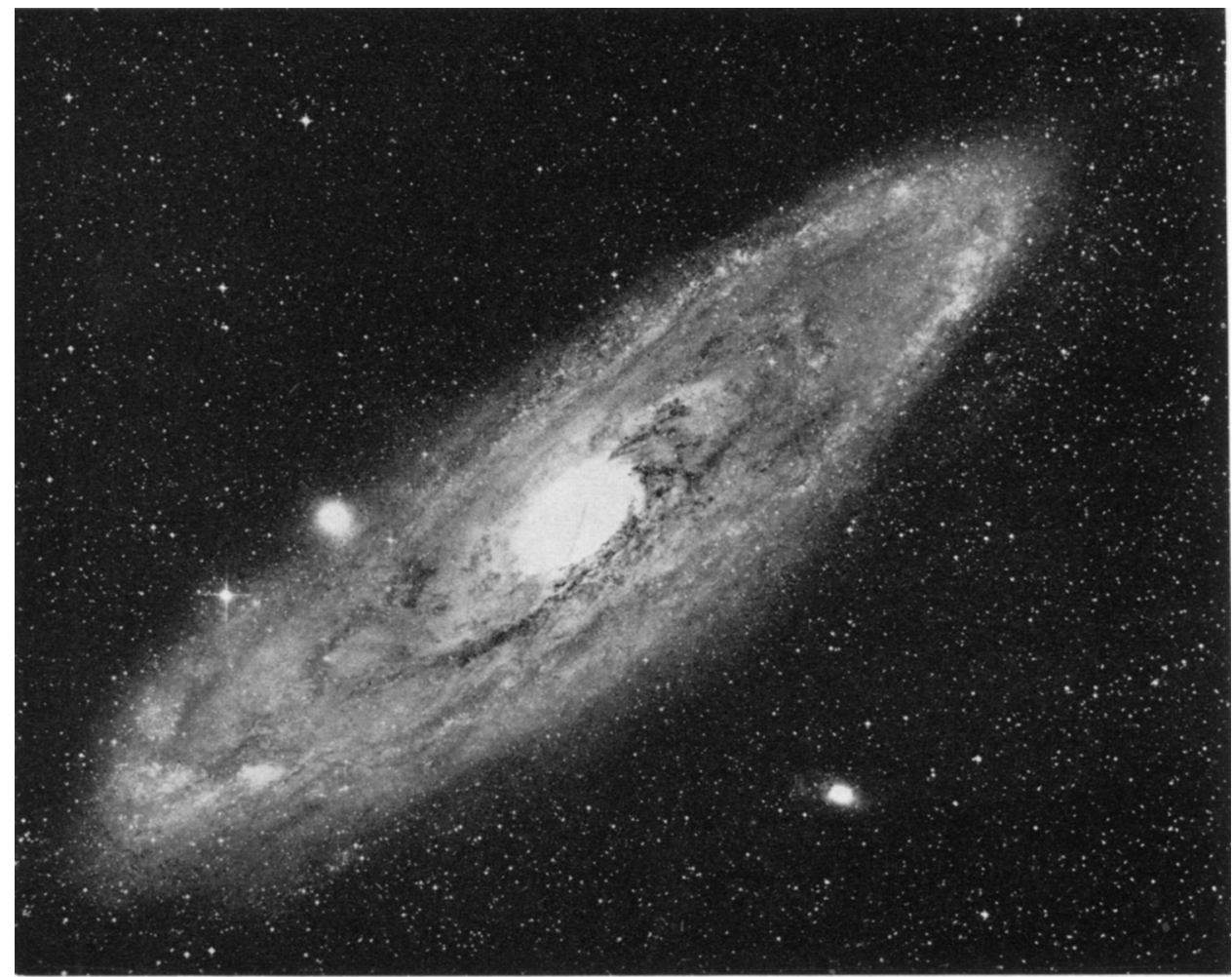

Fig. 30c.

Figs. 30a-c. The figures show the Crab Nebula (a), the large Magellanic Cloud (b) and M 31 (c) as they appear in visible light. The resolution that would be obtained in X-rays by use of the LOXT experiment is identical. The notes to the left of the figure show the properties of the Crab Nebula as they actually are and as they would appear if it were removed to the large Magellanic Clouds or the Andromeda Nebula.

\section{References}

Angel, J. R. P., and Weisskopf, M. C.: 1970, Astron. J. 75, 231.

Angel, J. R. P., Novick, R., VandenBout, P., and Wolff, R.: 1969, Phys. Rev. Letters $22,861$.

Blake, R. L., Chubb, T. A., Friedman, H., and Unzicker, A. E.: 1965, Astrophys. J. 1, 142.

Cornell, C. M., Grader, R. J., Harris, J. H., Hill, R. W., Rumble, R. P., and Salmi, D.S.: 1971, Rev. Sci. Instr. (to be published).

de Jager, C.: 1967, in Highlights of Astronomy presented at the XIII General Assembly of the IAU, 1967.

Egidi, A., Marconero, R., Pizella, G., and Sperli, F.: 1969, Rev. Sci. Instr. 40, 88.

Evans, K. and Pounds, K. A.: 1968, Astrophys. J. $152,319$.

Fisher, P. C. and Meyerott, A. J.: 1966, IEEE Trans. Nucl. Sci. NS-13-580.

Giacconi, R. and Rossi, B.: 1960, J. Geophys. Res. 65, 773.

Giacconi, R. and Rossi, B.: 1961, U.S. Patent 3, 143, 651, filed February 23.

Giacconi, R., Reidy, W. P., Zehnpfennig, T., Lindsay, J. C., and Muney, W. S.: 1965 Astrophys. J. 142, 1274.

Giacconi, R., Gorenstein, P., Gursky. H., Kellogg, E. M., and Tananbaum, H.: 1968a. 'Th' X-r $(1)^{\prime}$ Explorer Experiment, ASE-2149. 
Giacconi, R., Gursky, H., and Van Speybroeck, L. P.: 1968b, Ann. Rev. Astron. Astrophys. 6, 373.

Giacconi, R., Reidy, W. P., Vaiana, G. S., Van Speybroeck, L. P. and Zehnpfennig, T. F.: 1969, Space Sci. Rev. 9 3-57.

Goodrich, G. W. and Wiley, W. C.: 1962, Rev. Sci. Instr. 33, 761.

Gorenstein, P., Harris, B., Gursky, H., and Giacconi, R.: 1971, this volume, p. 183.

Gursky, H.: 1970, in L. Gratton (ed.) 'Non-Solar X- and Gamma-Ray Astronomy', IAU Symp. 37, 5.

Gursky, H., Giacconi, R., Gorenstein, P., Waters, J. R., Oda, M., Bradt, H., Garmire, G., and Sreekantan, B. V.: 1966, Astrophys. J. 144 and 146, 317.

Kirkpatrick, P. and Baez, A. V.: 1948, J. Opt. Soc. Am. 38, 766.

Kitamura, T., Migamoto, S., Nakagawa, M., and Takagishi, T.: 1969, Space Res. 9, North-Holland Publ. Co., Amsterdam.

Mathieson, E. and Sanford, P. W.: Proc. Intern. Symp. Nucl. Electron. Paris, p. 65.

Mertz, L.: 1968, Proceeding of the Symposium on Modern Optics, Polytechnic Institute of Brooklyn, March 1967.

Neupert, W. M., Gates, W., Swartz, M., and Young, R.: 1967, Astrophys. J. Letters 149, L79.

Oda, M.: Appl. Opt. 4, 143.

Oda, M., Bradt, H., Garmire, G., Spada, G., Sreekantan, B. V., Gursky, H., Giacconi, R., Gorenstein, P., and Waters, J. R.: Astrophys. J. 1967, 148, L5.

Schnopper, H. W. and Kalata, K.: 1969a, Applied. Phys. Letters 15, 134.

Schnopper, H. W. and Kalata, K.: 1969b, Astron. J. 74, 854.

Schnopper, H. W., Thompson, R. I., and Watt, S.: 1968, Space Sci. Rev. 8 534-42.

Schnopper, H. W., Bradt, H. V., Rappaport, S., Bengdran, E., Burnett, B., Doxsey, R., Mayer, W., and Watt, S.: 1970, to be published.

Underwood, J. H. and Muney, W. S.: 1967, Solar Phys. 1, 129.

Vaiana, G. S., Reidy, W. P., Zehnpfennig, T., Van Speybroeck, L., and Giacconi, R.: 1968, Science $161,465$.

Walker, A. B. C., Rugge, H. R., Chater, W. T., and Howey, C. K.: 1967, Trans. Am. Geophys. Union 48, 151 .

Wolber, W. G., Klettke, B. D., and Lintz, H. K.: 1969, Rev. Sci. Instr. 40, 1364.

Wolter, H.: 1952, Ann. Physik 10, 94 and 286.

X-ray Astronomy Groups at AS\&E, Columbia University, Goddard Space Flight Center, and MIT, -

Preliminary Study: 'Telescopes and Scientific Subsystems for a High Energy Astronomy Observatory”; 1 August 1969, Revised 26 September 1969, ASE-2266-A. 